\title{
Topological Order and Conformal Quantum Critical Points
}

\author{
Eddy Ardonne,, , Paul Fendley, ${ }^{2, \text { 由 }}$ and Eduardo Fradkin 1 , \\ ${ }^{1}$ Department of Physics, University of Illinois at Urbana-Champaign, \\ 1110 W. Green St. , Urbana, IL 61801-3080, USA \\ ${ }^{2}$ Department of Physics, University of Virginia, Charlottesville, VA 22901-4714, USA
}

(Dated: October 22, 2018)

\begin{abstract}
We discuss a certain class of two-dimensional quantum systems which exhibit conventional order and topological order, as well as two-dimensional quantum critical points separating these phases. All of the ground-state equal-time correlators of these theories are equal to correlation functions of a local two-dimensional classical model. The critical points therefore exhibit a time-independent form of conformal invariance. These theories characterize the universality classes of two-dimensional quantum dimer models and of quantum generalizations of the eight-vertex model, as well as $\mathbb{Z}_{2}$ and non-abelian gauge theories. The conformal quantum critical points are relatives of the Lifshitz points of three-dimensional anisotropic classical systems such as smectic liquid crystals. In particular, the ground-state wave functional of these quantum Lifshitz points is just the statistical (Gibbs) weight of the ordinary 2D free boson, the 2D Gaussian model. The full phase diagram for the quantum eight-vertex model exhibits quantum critical lines with continuously-varying critical exponents separating phases with long-range order from a $\mathbb{Z}_{2}$ deconfined topologically-ordered liquid phase. We show how similar ideas also apply to a well-known field theory with non-Abelian symmetry, the strong-coupling limit of $2+1$-dimensional Yang-Mills gauge theory with a Chern-Simons term. The ground state of this theory is relevant for recent theories of topological quantum computation.
\end{abstract}

\section{INTRODUCTION}

During the past decade and a half there has been an intense search for new kinds of theories describing quantum condensed-matter systems. Many experimental results have implied that stronglycorrelated fermionic systems exhibit qualitatively new types of physical behavior. The now-classic example of this is the fractional quantum Hall effect, where one of the striking consequences of strong correlations is that the Laughlin quasiparticles have fractional charge and fractional statistics, even though though the microscopic degrees of freedom are electrons with integer charge and fermionic statistics [1].

Traditionally one classifies different phases in terms of order parameters which give a global characterization of the physical state. In turn, the local fluctuations of this order parameter field drive the phase transitions between ordered and disordered states of these systems. This viewpoint, pioneered by Landau and his school, has been extremely successful in condensed matter physics and in other areas of physics, such as particle physics, through the powerful underlying concept of spontaneous symmetry breaking. Much of the structure of modern theory of critical phenomena is based on this point of view [2,3].

However, there are many different experimentally-realizable phases (and even more realizable theoretically!) in the fractional quantum Hall effect, but no local order parameter distinguishes between them. These phases are incompressible liquid states which have a fully gapped spectrum and do not break any symmetries of the Hamiltonian. The lack of a local order parameter led to many interesting discussions of the off-diagonal long-range order in the Hall effect [4]. One particularly elegant way of characterizing the order in the fractional quantum Hall effect is as topological order [5]. The topological order parameters are non-local; they are expectation values of operators which are lines or loops. Because of this, they can (and do) depend on topology: their value depends on the genus of the twodimensional surface on which the electrons live. One interesting characteristic of a topological phase is that the correlation functions in the ground state do not depend on the locations of the operators, but 
only on how the loops braid through each other. In addition, the degeneracies of these non-symmetry breaking ground states on topologically non-trivial manifolds are determined by the topology of these manifolds [6].

Although so far the only unambiguous experimental realizations of topological phases are in the fractional quantum Hall effect, there has been considerable effort to find, both theoretically and experimentally, condensed matter systems whose phase diagrams may exhibit topological ground states. Much of the current work involves studying fractionalized phases in time-reversal invariant systems (see e.g. 7 8]). One reason is that the "normal state" of high-temperature superconductors lacks an electron-like quasiparticle state in its spectrum. There are reasons to believe that frustrated magnets may also exhibit fractionalized behavior as well.

A particularly well-known and simple model with a topological phase is the quantum dimer model, which was invented as a way of modeling the short-range resonating-valence-bond theory of superconductivity [9]. The degrees of freedom of this two-dimensional model are classical dimers living on a two-dimensional lattice. With a special choice of Hamiltonian (called the RK point), the exact ground-state wave function can be found [10]. When the dimers are on the square lattice, the result is a critical point. If one deforms this special Hamiltonian, one generically obtains ordered phases. However, a topological phase occurs in the quantum dimer model on the triangular lattice [8]. When the quantum dimer model is in a topological phase, an effect analogous to fractionalization occurs [9]. This is called spin-charge separation. One can view the dimers as being created by nearest-neighbor pairs of lattice electrons in a spin-singlet state. Even though the fundamental degrees of freedom (the electrons) have both spin and charge, one finds that the basic excitations have either charge (holons) or spin (spinons), but not both. To prove this occurs, one must show that if one breaks apart an electron pair (dimer) into two holons or two spinons, they are deconfined. For the triangular-lattice quantum dimer model, this was shown in Ref. 8]; the analogous statement in terms of holon-holon correlators was proven in Ref. 11]. At a quantum critical phase transition between an ordered/confining phase and a disordered/deconfining phase (or between different confining states), confinement is lost: the RK point is deconfining 12, 13. 14 .

The notion of spin-charge separation is one of the basic assumptions behind the RVB theories of high-temperature superconductivity 9, 15, 16, 17, 19, 20, 21, 22], which effectively can be regarded as strongly-coupled lattice gauge theories. In $2+1$-dimensional systems spin-charge separation can only take place if these gauge theories are in a deconfined phase [13,23,24]. In $2+1$-dimensions this is only possible for discrete gauge symmetries. For a continuous gauge group, say $U(1)$ or $S U(2), 2+1$ dimensional gauge theories are always in a confining phase, unless the matter fields carry a charge higher than the fundamental charge so that the gauge symmetry is broken to a discrete subgroup [25]. Thus, the only consistent scenarios for spin-charge separation necessarily involve an effective discrete gauge symmetry, which in practice reduces to the simplest case $\mathbb{Z}_{2}$. Of particular interest is the fact that the low-energy sector of the deconfined phases of discrete gauge theories are the simplest topological field theories $26,27,28]$.

Many of these ideas have their origin in the conceptual description of confined phases of gauge theories as monopole condensates, and of their deconfined states as "string condensates" [29]. In gauge theories it has long been known that their phases cannot characterized by a local order parameter, since local symmetries cannot be spontaneously broken. The phases of gauge theories are understood instead in terms of the behavior of generally non-local operators such as Wilson loops and disorder operators 30.31], a concept borrowed from the theory of the two-dimensional Ising magnet 32].

Interesting as they are, the applicability of these ideas to the problem of high-temperature superconductivity and other strongly correlated systems is still very much an open problem. Topological fractionalized ground states are not the only possible explanation of the unusual physics of the cuprates. In fact, when constructing local microscopic models of strongly-correlated systems which are suspected to have fractionalized phases, many theorists have found that instead these models have a strong tendency to exhibit spatially-ordered states, a.k.a. "valence bond crystals", which appear to compete 
with possible deconfined states. It is now clear that the regimes of strongly-correlated systems which may favor fractionalized phases also favor, and perhaps more strongly, non-magnetic spatially ordered states of different types, including staggered flux states [17], or $d$-density wave states [33], and electronic analogs of liquid crystalline phases [34,35]. By now there are a number of examples of models with short-range interactions whose phase diagrams contain both fractionalized and spatially-ordered phases [8,36, 37, 38]. It has recently been proposed that deconfined critical points may describe the quantum phase transitions between ordered Néel states and valence bond crystals [39].

For several reasons, most of the studies of topological order and quantum critical points have focused on examples with two spatial dimensions. The experimental reason is that the Hall effect is two-dimensional, and typical strongly-correlated systems, such as the cuprate high-temperature superconductors, are often effectively two-dimensional. Theoretically, it is because in two dimensions particles can have exotic statistics interpolating between bosonic and fermionic [40]. A common characteristic of topological phases in two dimensions is the presence of exotic statistics, which occur in the fractional quantum Hall effect [4]. The statistics can even be non-abelian: in some cases, the change in the wave function depends on the order in which particles are exchanged [41]. Systems with non-abelian statistics are particularly interesting because they are useful for error correction in quantum computers $42,43,44,45,46]$.

In this paper we will discuss models with topological phases and ordered phases, as well as quantum phase transitions separating them. There has also been a great deal of interest in quantum critical points in and of themselves [47]. At a quantum critical point, the physics is of course scale invariant, but it need not be Lorentz invariant. The quantum critical points discussed in this paper have dynamical critical exponent $z=2$, instead of the usual $z=1$ of a Lorentz-invariant theory. This allows for some striking new physics. The action of these $z=2$ quantum critical points is invariant under time-independent conformal transformations of the two-dimensional space. A remarkable consequence is that the ground-state wave functionals of the field theories discussed here are conformally invariant in space. This means that the ground state wave functional is invariant under any angle-preserving coordinate transformations of space. For two-dimensional space, there is an infinite set of such transformations, as is familiar from studies of two-dimensional conformal field theory [48]. This sort of behavior is not common at all: the action of a field theory at a critical point is often scale invariant (and also conformally invariant), but the ground-state wave functional itself in general is not. We dub critical points with this behavior conformal quantum critical points.

One of the consequences of the conformal invariance of the ground state wave function is that all the equal-time correlators of the quantum theory are equal to suitable correlation functions of observables of a two-dimensional Euclidean conformal field theory. We will exploit this connection in this paper quite extensively. However, just as important, conformal invariance of the wave function implies that the ground state of this $2+1$-dimensional theory at a conformal quantum critical point must have zero resistance to shear stress in the two-dimensional plane.

This can be seen as follows. Consider an infinitesimal local distortion of the geometry of the two-dimensional plane represented by an infinitesimal change $\delta g_{i j}(x)$ of the two-dimensional metric, as is conventional in the theory of elasticity $[2]^{1}$. Let $|\Psi\rangle$ be the ground state wave function for the undistorted plane and $|\Psi(g)\rangle$ be the ground state wave function in the distorted plane with twodimensional metric $g_{i j}(x)=\delta_{i j}+\delta g_{i j}(x)$. Under this distortion the Hamiltonian of the system changes by an amount

$$
\delta H(g)=\int d^{2} x \frac{\delta H}{\delta g_{i j}(x)} \delta g_{i j}(x)+\ldots
$$

\footnotetext{
${ }^{1}$ Recall that the change in the metric, given by the strain tensor, is quadratic in the local deformation of the system.
} 
To first order in perturbation theory in $\delta H$, the change of the ground state wave energy is

$$
\delta E_{0}=\frac{\langle\Psi|\delta H(g)| \Psi\rangle}{\langle\Psi \mid \Psi\rangle} \equiv\langle\delta H(g)\rangle=\int d^{2} x\left\langle\frac{\delta H}{\delta g_{i j}(x)}\right\rangle \delta g_{i j}(x)+\ldots
$$

where $E_{0}$ is the exact ground state energy of the distorted system. On the other hand, the change of the norm of the ground state wave function $\|\Psi\|$ is, to all orders in perturbation theory, given by [49]

$$
\|\Psi\|^{2}=\frac{\partial E_{0}}{\partial \varepsilon_{0}}
$$

where $\varepsilon_{0}$ is the ground state energy of the undistorted system. Thus, the change of the norm $\|\Psi\|$ is determined by the $(2 \times 2)$ stress tensor $T_{i j}$ of the $2+1$-dimensional theory

$$
T_{i j}(x)=\left\langle\frac{\delta H}{\delta g_{i j}(x)}\right\rangle \text {. }
$$

On the other hand, we can regard $\|\Psi\|^{2}$ as the partition function $Z$ of a two-dimensional Euclidean conformal field theory. This theory has an Euclidean stress-energy tensor, $T_{i j}^{\mathrm{cft}}$, defined by [48]

$$
T_{i j}^{\mathrm{cft}}=-\left\langle\frac{\delta S_{\mathrm{cft}}}{\delta g_{i j}(x)}\right\rangle=\frac{\delta \ln Z}{\delta g_{i j}(x)}
$$

which essentially coincides with the stress tensor of the $2+1$-dimensional quantum field theory defined above. Scale invariance, rotational invariance and conservation require that $T_{i j}$ be a conserved (divergence free) symmetric traceless tensor. Consequently, the effective Hamiltonian (as well as the action) at this quantum critical point can depend on the spatial gradients of the field only through the "spatial curvature", e.g. $\left(\nabla^{2} \varphi\right)^{2}$ in a scalar field theory. In other words, at a conformal quantum critical point for a scalar theory, the stiffness vanishes: the usual $(\nabla \varphi)^{2}$ term is not possible. This means that the dynamical critical exponent of this quantum critical theory must be $z=2$. We call such theories quantum Lifshitz theories; we will discuss such critical points in detail.

In this paper we discuss lattice models which exhibit both ordered/confined phases and disordered/deconfined phases. We will also discuss the field-theory description of these phases and of the phase transitions. To simplify matters, and to be able to obtain exact results, we will introduce models whose ground-state wave function will be known exactly and whose properties we will be able to determine quite explicitly. In this sense, these models are a generalization of the quantum dimer model at the RK point. The basis of the Hilbert space of these models is the configuration space of a two-dimensional classical statistical-mechanical system or Euclidean field theory. Each of these basis states is defined to be orthogonal with respect to the others. An arbitrary state in this Hilbert space can therefore be described as some linear combination of these basis elements. Describing the Hilbert space in such a fashion is not particularly novel. The unusual feature of the models we will discuss is that the ground-state wave function can be expressed as in terms of the action or Boltzmann weights of a local two-dimensional classical theory. The normalization of the wave function will then be the partition function or functional integral of the classical two-dimensional model. This special property is why the wave functionals at the critical points are have a time-independent conformal invariance at their critical points. The field theory of these conformal quantum critical points can be extended to describe nearby ordered and disordered phases, including their confinement properties. We will study this quite explicitly in a quantum generalization of the eight-vertex model. However, much of the physics we discuss should apply to topological phases and $(z=2)$ quantum critical points in general.

We will also study theories with a continuous non-abelian symmetry. We show that, interestingly enough, it is very difficult to construct a non-trivial conformal quantum critical point with such a symmetry. We do find a Hamiltonian whose ground state is the doubled Chern-Simons theory of Ref. 
50 51]. This is a time-reversal invariant theory of interest in topological quantum computation and in (ordinary) supercondutivity; it is in a gapped topological phase.

In section [1] we discuss the simplest model with a scale-invariant critical wave function, the quantum dimer model at the RK point. Here we also introduce the quantum Lifshitz model, the effective field theory of these new quantum critical points. In section III we generalize the relation between the quantum dimer model and the scalar field theory discussed in section II to include perturbations which drive the system in to a quantum disordered/deconfined phase or to a ordered/confined phase. In section [V] we define the quantum eight-vertex model by finding a Hamiltonian whose ground-state wave function is related to the classical eight-vertex model. This will allow us to find quantum critical lines with variable critical exponents separating a $\mathbb{Z}_{2}$-ordered phase from a topologically-ordered phase. It will also allow us to place a number of previously-known models, in particular that of Ref. [42], in a more general setting. We show in detail how to use the known results from the Baxter solution of the classical model to map out the critical behavior of the quantum theory. In particular we analyze in detail the confinement and deconfinement properties of the different phases and at criticality. In section $\mathrm{V}$ we study the non-abelian case, and see that the strongly-coupled limit of Yang-Mills theory with a Chern-Simons term has a wave functional local in two-dimensional classical fields [52]. This theory is in a phase with topological order. In three appendices we give details of the correlators of the quantum Lifshitz field theory (Appendix $\mathrm{A}$ ), and of the gauge-theory construction of the quantum six-vertex (Appendix [B) and eight-vertex (Appendix [C) models.

\section{SCALE-INVARIANT WAVE FUNCTIONS AND QUANTUM CRITICALITY}

The simplest lattice model we discuss is the quantum dimer model; the simplest field theory we dub the quantum Lifshitz theory. They both provide very nice illustrations of the properties discussed in the introduction. In the quantum dimer model, the space of states consists of close-packed hard-core dimers on a two-dimensional lattice. A quantum Hamiltonian therefore is an operator acting on this space of dimers, taking any dimer configuration to some linear combination of configurations. In every configuration exactly one dimer must touch every site, so any off-diagonal term in the Hamiltonian must necessarily move more than one dimer. The simplest such operator is called a "plaquette flip": if one has two dimers on opposite sites of one plaquette, one can rotate the dimers around the plaquette without effecting any other dimers. For example, for the $i$ th plaquette on the square lattice one has

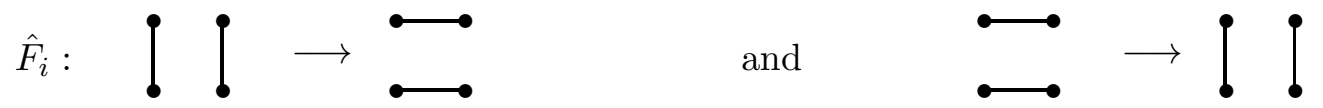

The operator $\hat{F}_{i}$ is defined as zero on any other dimer configuration around a plaquette (i.e. if the $i$ th plaquette is not flippable). We define the operator $\hat{V}_{i}$ as the identity if the plaquette is flippable, and zero otherwise.

The Rokhsar-Kivelson Hamiltonian for the quantum dimer model [10]

$$
H_{R K}=\sum_{i}\left(\hat{V}_{i}-\hat{F}_{i}\right)
$$

has the remarkable property that one can find its ground states exactly. They have energy zero, and every state (in a given sub-sector labeled by global conserved quantities) appears with equal amplitude in its ground-state wave function. These properties follow from the facts that $H_{R K}$ is self-adjoint, and $\left(\hat{V}_{i}-\hat{F}_{i}\right)^{2}=2\left(\hat{V}_{i}-\hat{F}_{i}\right)$. Hamiltonians of the form $H=\sum_{i} Q_{i}^{\dagger} Q_{i}$ necessarily have eigenvalues $E$ obeying $E \geq 0$. Moreover, if one can find a state annihilated by all the $Q_{i}$, then it is necessarily a ground state. The equal-amplitude sum over all states is indeed such a state. In the Schrödinger picture, the wave function for this state is easy to write down. Define $Z$ as the number of all dimer configurations 
in some finite volume. This is precisely the classical partition function of two-dimensional dimers with all configurations weighted equally. Then the properly-normalized ground-state wave function for any basis state $|C\rangle$ in the Hilbert space (i.e., any classical dimer configuration $C$ ) is

$$
\left|\Psi_{0}\right\rangle=\frac{1}{\sqrt{Z}} \sum_{C}|C\rangle \Rightarrow \Psi_{0}(C)=\frac{1}{\sqrt{Z}}
$$

The wave function of the quantum system is indeed related to the classical system.

One can extend this sort of analysis to compute equal-time correlators in the ground state. One finds simply that these correlators are given by the correlation functions of the two-dimensional classical theory. Thus for $H_{R K}$ for dimers on the square lattice, one finds algebraic decay of the correlation functions 10]. This model is then interpreted as a critical point between two ordered phases of the dimers [13,23]. However, for the analogous Hamiltonian on the triangular lattice, the classical twodimensional correlators are exponentially decaying [8]. One can also show that spinon-type excitations (sites without a dimer) are deconfined on the triangular lattice [11]. This means the quantum dimer model with $H_{R K}$ on the triangular lattice is interpreted as being in a "liquid" phase, which has a mass gap and exponential decay of interactions, but which has no non-zero local order parameter.

Such a relation between two-dimensional quantum theories is not limited to lattice models, nor are the ground-state wave functions required to be equal-amplitude sums over all configurations. We will construct now a simple (non-Lorentz invariant) two-dimensional quantum critical field theory i.e., a theory whose ground state wave function represents a two-dimensional conformal theory.

Consider a free boson $\varphi(x, t)$ in two spatial dimensions and one time dimension. Instead of the usual Hamiltonian quadratic in derivatives, we use one which has been conjectured by Henley [53] to belong to the same universality class as the square-lattice quantum-dimer model. It is

$$
H=\int d^{2} x\left[\frac{\Pi^{2}}{2}+\frac{\kappa^{2}}{2}\left(\nabla^{2} \varphi\right)^{2}\right]
$$

where $\Pi=\dot{\varphi}$ as usual. The associated Euclidean action for the field $\varphi$ is

$$
S=\int d^{3} x\left[\frac{1}{2}\left(\partial_{\tau} \varphi\right)^{2}+\frac{\kappa^{2}}{2}\left(\nabla^{2} \varphi\right)^{2}\right]
$$

This system, Eq. (2.5), also arises in three-dimensional classical statistical mechanics in the field-theory description of Lifshitz points 2], for example in (smectic) liquid crystals. For this reason we will call the system with Hamiltonian (2.4) the quantum Lifshitz model. Of particular relevance to our discussion is the long-ago observation by Grinstein [54] that this system is analogous to the two-dimensional Euclidean free boson in that it represents a line of fixed points parametrized by $\kappa$.

Let us rederive this result by quantizing the Hamiltonian (2.4). We impose the canonical commutation relations

$$
\left[\varphi(\vec{x}), \Pi\left(\vec{x}^{\prime}\right)\right]=i \delta\left(\vec{x}-\vec{x}^{\prime}\right)
$$

so in the Schrödinger picture the canonical momentum is the functional derivative $\Pi(\vec{x})=-i \delta / \delta \varphi(\vec{x})$. The Schrödinger equation for the wave functional $\Psi[\varphi]$ is then

$$
\int d^{2} x\left[-\frac{1}{2}\left(\frac{\delta}{\delta \varphi}\right)^{2}+\frac{\kappa^{2}}{2}\left(\nabla^{2} \varphi\right)^{2}\right] \Psi[\varphi]=E \Psi[\varphi] .
$$

We can find the ground-state wave function in the same fashion as we did for $H_{R K}$. Indeed, if we define

$$
Q(x) \equiv \frac{1}{\sqrt{2}}\left(\frac{\delta}{\delta \varphi}+\kappa \nabla^{2} \varphi\right), \quad Q^{\dagger}(x) \equiv \frac{1}{\sqrt{2}}\left(-\frac{\delta}{\delta \varphi}+\kappa \nabla^{2} \varphi\right)
$$


the (normal-ordered) quantum Hamiltonian is then

$$
H=\frac{1}{2} \int d^{2} x\left\{Q^{\dagger}(\vec{x}), Q(\vec{x})\right\}-\varepsilon_{\mathrm{vac}} V \equiv \int d^{2} x Q^{\dagger}(\vec{x}) Q(\vec{x})
$$

which is Hermitian and positive. Here $V$ is the spatial volume (area) of the system, and we have normal-ordered the Hamiltonian by subtracting off the (UV divergent) zero-point energy density

$$
\varepsilon_{\mathrm{vac}}=-\frac{\kappa}{2} \lim _{\vec{y} \rightarrow \vec{x}} \nabla_{x}^{2} \delta(\vec{x}-\vec{y})>0
$$

Any state annihilated by $Q(x)$ for all $x$ must be a zero-energy ground state. The corresponding groundstate wave functional $\langle[\varphi] \mid v a c\rangle=\Psi_{0}[\varphi]$ satisfies $Q \Psi_{0}[\varphi]=0$, where $Q$ is defined in Eq. (2.8). This is simply a first-order functional differential equation, and is easily solved, giving

$$
\Psi_{0}[\varphi]=\frac{1}{\sqrt{\mathcal{Z}}} e^{-\frac{\kappa}{2} \int d^{2} x(\nabla \varphi(x))^{2}}
$$

where $\mathcal{Z}$ is the normalization

$$
\mathcal{Z}=\int[\mathcal{D} \varphi] e^{-\kappa \int d^{2} x(\nabla \varphi)^{2}}
$$

The probability of finding the ground state in the configuration $|[\varphi]\rangle$ is therefore

$$
\left|\Psi_{0}[\varphi]\right|^{2}=\frac{1}{\mathcal{Z}} e^{-\kappa \int d^{2} x(\nabla \varphi)^{2}}
$$

Consequently, the ground state expectation value of products of Hermitian local operators $\mathcal{O}[\varphi(\vec{x})]$ reduces to expressions of the form

$$
\left\langle\operatorname{vac}\left|\mathcal{O}\left[\varphi\left(\vec{x}_{1}\right)\right] \ldots \mathcal{O}\left[\varphi\left(\vec{x}_{n}\right)\right]\right| \operatorname{vac}\right\rangle=\frac{1}{\mathcal{Z}} \int[\mathcal{D} \varphi] \mathcal{O}\left[\varphi\left(\vec{x}_{1}\right)\right] \ldots \mathcal{O}\left[\varphi\left(\vec{x}_{n}\right)\right] e^{-\kappa \int d^{2} x(\nabla \varphi)^{2}}
$$

This two-dimensional quantum theory has a deep relation with a two-dimensional classical theory: the ground-state expectation value of all local observables are mapped one-to-one to correlators of a two-dimensional massless Euclidean free boson. The latter is a well-known conformal field theory, and its correlation functions are easily determined (for convenience we give them explicitly in Appendix A). This two-dimensional critical field theory is conformally-invariant, so the equal-time correlators of the quantum theory must reflect this. This scalar field theory is therefore not only quantum critical but it also has a time-independent conformal invariance.

The equal-time expectation values of the "charge operators" $\mathcal{O}[\varphi]$, as well as the correlation functions of the dual vortex (or "magnetic") operators discussed in Appendix A exhibit a power-law behavior as a function of distance, as expected at a quantum critical point. As shown also in Appendix A their autocorrelation functions also exhibit scale invariance albeit with a dynamic critical exponent $z=2$. This behavior of the equal-time correlator was shown earlier to occur in the quantum dimer model on the square lattice at the RK point: there is a massless "resonon" excitation, and the equal-time correlation functions for two static holons has a power law behavior equal to that of the monomer correlation function in the classical 2D dimer model on the square lattice.

However, not all theories whose ground state can be found in this fashion need be critical with power-law behavior. As noted above, the quantum dimer model on the triangular lattice is not. As 
we will discuss in section III, adding say a mass-like term to $Q(x)$ in the scalar field theory gives a theory with exponentially-decaying correlations in the ground state. Thus one can understand phase transitions in such theories as well, and we will explore several of these in this paper. Nevertheless, we expect that when the quantum phase transition is continuous, the quantum critical points of generic theories of this type will have the basic structure of the quantum Lifshitz model. As discussed in the introduction, only quantum Lifshitz points can be conformal quantum critical points. ${ }^{2}$

In the next sections we will show that generalizations of this $z=2$ quantum Lifshitz Hamiltonian also describe the quantum phase transitions between generalizations of the valence bond crystal states and quantum disordered states which describe deconfined topological fluid phases (provided these quantum phase transitions are continuous). Notice that phase transitions from deconfined to confined, uniform and translationally-invariant states are described by the standard Lorentz invariant $z=1$ critical point of gauge theories [25, 31, 57].

These examples show that one can obtain precise information about some $2 \mathrm{~d}$ quantum systems in terms of known properties of $2 \mathrm{~d}$ classical systems. The trick of doing so is in finding a set of $Q_{i}$ (or $Q(x)$ in the continuum) which annihilates the equal-amplitude state (or some other desired state), and then defining $H=\sum_{i} Q_{i}^{\dagger} Q_{i}[58]$. This seems like it should be possible to do for any classical $2 \mathrm{~d}$ theory, and indeed, there are many known examples of this sort. However, it is not clear for a given $2 \mathrm{~d}$ classical theory one can always find a Hamiltonian which is both local and ergodic (in this context, ergodic means that the Hamiltonian will eventually take the system through all of phase space with a given set of conserved quantum numbers). It is also not clear that even if such a Hamiltonian exists, whether it will have any physical relevance.

Moreover, this simple relation of the ground-state wave function of a $2 \mathrm{~d}$ quantum system to a $2 \mathrm{~d}$ classical system is not at all generic: the quantum dimer model with $H_{R K}$ and this quantum Lifshitz field theory are quite special. To illustrate this, let us discuss briefly the ground-state wave functions of standard quantum field theories at a (quantum) critical point. Consider first the most common case, the Lorentz invariant $\varphi^{4}$ field theory at criticality. Below $D=4$ space-time dimensions this critical theory is controlled by its non-trivial Wilson-Fisher fixed point. The resulting theory is massless and in general it has an anomalous dimension $\eta \neq 0$. Scale and Lorentz invariance fully dictate the behavior of all the correlation functions at this fixed point. General fixed point theories are scale invariant and, in addition, they exhibit an enhanced, generally finite-dimensional, conformal symmetry. It is a very special feature of $D=1+1$-dimensional Lorentz-invariant fixed point theories that they exhibit a much larger, infinite-dimensional, conformal invariance. This enhanced symmetry leads to a plethora of critical behaviors in $1+1$ dimensions. In contrast, there are relatively few known distinct critical points in higher dimensions for Lorentz-invariant field theories.

It is well known that the knowledge of all the equal-time correlation functions determines completely the form of the ground state wave function, i.e. in the Schrödinger representation of the field theory $[59$, 6061 . For a general theory, the ground-state wave function is a non-local and non-analytic functional of the field configuration. Thus, at the Wilson-Fisher fixed point, which describes theories with only a global conformal (scale) invariance, the structure of the ground state wave function is quite complicated. For instance, the probability of a constant field configuration $\varphi(\vec{x})=\varphi$ at a critical point has the universal form 61 ]

$$
\left|\Psi_{\mathrm{vac}}(\varphi)\right|^{2}=A e^{-B|\varphi|^{1+\delta}}
$$

For a Lorentz-invariant $\varphi^{4}$ theory the universal critical exponent is given by $\delta=(d+2-\eta) /(d-2+\eta)$, and $A$ and $B$ are two non-universal constants. Therefore, at criticality the wave function in general is a non-analytic non-local functional of the field configuration. In contrast, the ground-state wave function

\footnotetext{
2 The role of conformal invariance in $2 \mathrm{D}$ classical dynamics with $z=2$ and anisotropic 3D classical Lifshitz points was
} considered recently in Refs. 55 [56]. 
of a 1+1-dimensional relativistic interacting fermions (a Luttinger liquid), which is a conformal field theory, has a universal non-local non-analytic Jastrow-like power law factorized form 62] consistent with the form found by the Bethe-ansatz solution of the Calogero-Sutherland model [63, 64, 65]. This structure is a consequence of the (local) conformal invariance of the $1+1$-dimensional theory. Even in $1+1$ dimensions, the wave function is generically non-local.

\section{DIMERS, FERMIONS AND THE QUANTUM LIFSHITZ FIELD THEORY}

In Section II we showed how to find the exact ground-state wave functions of the quantum dimer model with Rokhsar-Kivelson Hamiltonian $H_{R K}$, and the quantum Lifshitz scalar field theory. In this section, we will describe their properties in more detail, and some simple generalizations.

\section{A. From the square to the triangular lattice}

The ground states of $H_{R K}$ are the sum over all classical dimer configurations in a sector with equal amplitudes [10]. There are a number of useful generalizations to models where the ground state is still a sum over all states in a sector, but not necessarily with equal amplitudes. One interesting case is a quantum dimer model which interpolates between the square and triangular lattices.

A triangular lattice can be made from a square lattice by adding bonds across all the diagonals in one direction. The classical dimer model on the square lattice can be deformed continuously into the triangular-lattice model by assigning a variable weight $w$ for allowing dimers along these diagonals: for $w=0$ we have the original square-lattice model, while $w=1$ gives the triangular-lattice one [11]. There is a two-dimensional quantum Hamiltonian which has the $w$-dependent classical dimer model as its ground state. For dimers on opposite sides of a plaquette of the original square lattice, the Hamiltonian remains $H_{R K}$. In addition, however, parallel dimers on adjacent diagonals can also be flipped [8], see figure 1. Like the flip on the square lattice, this flip can be done without violating the
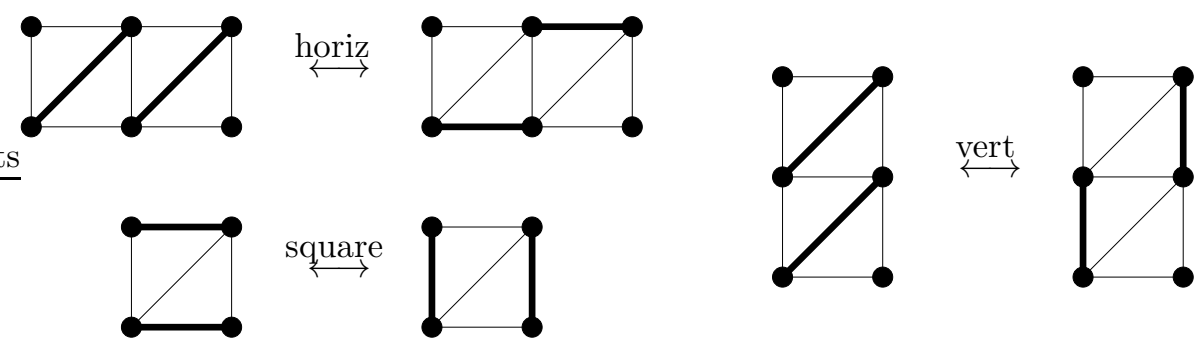

FIG. 1: Dimer flips on the triangular lattice

close-packing and hard-core constraints.

When the off-diagonal terms in the Hamiltonian $H_{w}$ consist solely of these flips, $H_{w}$ breaks up into two-by-two blocks like $H_{R K}$ does. In the ground state dimers along diagonals should get a weight $w$, so the Hamiltonian $H_{w}$ must explicitly depend on $w$. To construct a Hamiltonian with the desired ground state, we find a set of operators $Q_{i}$, each of which annihilates this state. For example, denote a configuration with dimers on adjacent diagonals as $|1\rangle$, and $|2\rangle$ as the configuration to which it is flipped, as shown in fig. 11 The $Q_{i}$ acting on these two configurations is then

$$
Q_{i}=\frac{1}{w^{2}+w^{-2}}\left(\begin{array}{cc}
w^{-2} & -1 \\
-1 & w^{2}
\end{array}\right)
$$


where the first row and column correspond to state $|1\rangle$, while the second corresponds to state $|2\rangle$. There are three types of $Q_{i}: Q_{i}^{\text {(square) }}$ acts on dimers on opposite sides of a plaquette of the original square lattice, while $Q_{i}^{\text {(horiz) }}$ and $Q_{i}^{\text {(vert) }}$ are associated to flips involving dimers on the 'diagonal' links; see figure 1. The operator $Q_{i}^{(\text {square) }}$ is given by (3.1) with $w=1$. We then take

$$
H_{w}=\sum_{i}\left[Q_{i}^{\text {(square) }}+w Q_{i}^{(\text {vert })}+w Q_{i}^{(\text {horiz })}\right]
$$

where the sum is over all plaquettes $i$. Each $Q_{i}$ is a projection operator, so $Q_{i}=Q_{i}^{\dagger}=\left(Q_{i}\right)^{2}$.

We have defined the operator $Q_{i}$ so that it annihilates the state $w^{2}|1\rangle+|2\rangle$. The ground state of $H_{w}$ is then the sum over classical dimer model states with each state weighted by $w^{\mathcal{D}}$, where $\mathcal{D}$ is the number of dimers along diagonals in that state. More precisely, one must find the conserved quantities for a given value of $w$ and boundary conditions; the sum over all states with weight $w^{\mathcal{D}}$ in that sector is an eigenstate of $H_{R K}$ with zero energy. The ground-state wave function for a configuration with $\mathcal{D}$ diagonal dimers is then

$$
\Psi_{0}[\mathcal{D}]=\frac{w^{\mathcal{D}}}{\sqrt{Z\left(w^{2}\right)}}
$$

where $Z(w)$ is the partition function for the classical dimer model with diagonal dimers receiving weight $w$. Note the $w^{2}$ in the argument of $Z$ in the denominator: this is because in quantum mechanics probabilities are given by $\left|\Psi_{0}\right|^{2}$. For the square or triangular lattice, this is unimportant, because $w=0$ or $w=1$, both of which have $w^{2}=w$. For $w=1$, we have the equal-amplitude sum over all dimer states of the triangular lattice, the model discussed in Ref. [8]. For $w=0$, we recover a slight generalization of the original square-lattice quantum-dimer model of Rokhsar and Kivelson [10]. In this limit, this Hamiltonian reduces to $H_{R K}$ plus a potential term forbidding dimers on adjacent diagonal links. Isolated diagonal dimers are still allowed for $w \rightarrow 0$, but since none of them can be flipped, the Hamiltonian does not affect them at all. Thus they can be viewed as fixed zero-energy defects in the square-lattice quantum-dimer model. The ground-state wave function for a given set of defects is the equal-amplitude over all configurations of dimers on the sites without defects.

Since we know the exact ground-state wave function for any $w$, one would like to compute the correlation functions in this model. In most two-dimensional lattice models, even those solvable by the Bethe ansatz, this is extraordinarily difficult or impossible. However, the classical dimer model is special in that one can do such computations, because like the two-dimensional Ising model, it is essentially free-fermionic. Precisely, its partition function and correlators can be written in terms of the Pfaffian (the square root of the determinant) of known matrices [66]. One can rewrite the Pfaffians in terms of a functional integral over Grassmann variables at every site on the lattice [67]. The action in the case of equal Boltzmann weights is quadratic in the Grassmann variables, so one can compute easily any ground-state correlation function using the dimers, because the dimers can be written in terms of the fermions. This was discussed for the triangular lattice in [8]. The correlators of spinon-like or holon-like excitations are much more complicated, but the computation was done for $w=0$ in [66], and for arbitrary $w$ in [11]. On the lattice, the holon is a defect or monomer, a site without a dimer. The holon-creation operators are not local in terms of the fermionic variables, in a manner reminiscent of how the spin and fermion operators are non-local with respect to each other in the two-dimensional Ising model. The holon two-point function is valuable in that it gives an order parameter for the phase with topological order: if it is non-vanishing as two holons are taken far apart, the holons are deconfined and we are indeed in a topological phase. The existence of topological order was previously established for the triangular lattice $w=1$ [8] 14]; in [11] the explicit correlator was computed, and indicates the topologically-ordered phase exists for any non-zero $w$.

The ground state of the quantum dimer model with $H_{w}$ is therefore well understood for any $w$. There are no exact results for the excited states, however. In fact, since $H_{w}$ does not have any action 
upon empty sites, one can give the holon any gap desired without changing the ground states. It is therefore useful to find a continuum limit and study the field theory describing this model. In other words, we would like to understand a field theory with partition function equal to the continuum limit of partition function $Z(w)$ in Eq. (3.3). Since ground-state correlators for the square lattice are algebraically decaying, the $w=0$ model is critical and should have a sensible continuum limit. Indeed, when $w=0$, the Grassmann variables turn into a single free massless Dirac fermion field; its action is the usual rotationally-invariant kinetic term. The dimer correlation length $\xi$ was computed exactly as a function of $w$; for the triangular lattice it is about one lattice spacing, while $\xi$ diverges as $1 / w$ for $w$ small [11]. Thus there is a field-theory description of the continuum limit of the classical dimer model valid as long as $w$ is scaled to zero with the lattice spacing $a$ such that $w / a$ remains finite. Since the action is still quadratic in the Grassmann variables, the resulting fermionic field theory remains free. However, the Dirac fermion receives a mass proportional to $w / a$ [1]].

There are several useful aspects of taking the continuum limit, apart from finding the excited-state spectrum. Correlators are easier to compute: for $w=0$ one can use conformal field theory [48], while in the scaling limit $w \rightarrow 0$ one can use form-factor techniques [68]. Another useful fact is that (ignoring boundary conditions), a Dirac fermion can be described in terms of two decoupled Ising field theories. In the continuum, the holon can be written in terms of the product of the spin field in one Ising model with the disorder field in the other Ising model. Taking $w$ away from zero amounts to giving one Ising order field an expectation value, and the other disorder field an expectation value. 11]. Thus one can see directly in continuum that the holon order parameter is non-vanishing for $w \neq 0$. We will see in the next section that one can also understand the physics of the quantum Lifshitz critical line for all $\kappa$ in terms of two (coupled) Ising models.

\section{B. The critical field theory}

We would therefore like to find a natural-looking quantum field-theory Hamiltonian which has as its ground-state wave functional

$$
\Psi_{0}[\psi]=\frac{e^{-S_{\text {Dirac }}[\psi]}}{\sqrt{Z_{\text {Dirac }}}}
$$

where $S_{\text {Dirac }}$ is the usual action for a rotationally-invariant action for a free Dirac fermion in two Euclidean dimensions. Since this wave functional involves Grassman numbers, the easiest way to think of $\left|\Psi_{0}\right|^{2}$ as a weight in the path integral defining all correlators. While it is possible to find a Hamiltonian acting on this fermionic basis, it is more convenient and more intuitive to instead use bosonic variables. It is more convenient because correlators in a massless Dirac fermion theory (including those involving the product of spin fields) can be bosonized, meaning that they can be written in terms of correlators of free scalar fields [48,69]. It is more intuitive because the classical dimer model on the square lattice has a simple description in terms of a "height" variable [53]. A height is an integer-valued variable, which typically in the continuum limit turns into a scalar field. This description will allow us also to make contact with the quantum Lifshitz model.

Recently, Moessner et al. [14] generalized Henley's argument of Ref. 53], and used the connection between quantum dimer models and their dual quantum roughening (height) models 12, 13, 23, 70, 72 73.74 to argue that the Hamiltonian of Eq. (2.4) actually defines the universality class of quantum critical points between valence bond crystal phases. The nature of the phase transition between valence bond crystal states is the focus of much current research. Quite recent results by Vishwanath et al. 75] ${ }^{3}$, and by Fradkin et al. [76], show that the transition between valence bond crystals is generically first order, as expected from a simple Landau argument. Nevertheless, when the transition is continuous,

\footnotetext{
${ }^{3}$ We became aware of Ref. [75] as this paper was being completed.
} 
it is described by the Hamiltonian of Eq. (2.4) which must be regarded as a (rather rich) multicritical point.

To see how the height description arises, let us go back to the square-lattice quantum dimer model, where $H_{w}$ reduces to $H_{R K}$. To map the square-lattice classical dimer model onto a height model, one first assigns a height variable to each plaquette. In going around a vertex on the even sub-lattice clockwise, the height changes by +3 if a dimer is present on the link between the plaquettes, and by -1 if no dimer is present on that link. On the odd sub-lattice, the heights change by -3 and +1 respectively. The flip operator $\hat{F}_{i}$ on a plaquette $i$ changes the height on that plaquette by either \pm 4 . To take the continuum limit, is convenient to turn this into a model with heights on the sites. We define $h$ on each site to be the average value of the four plaquette heights around that site ${ }^{4}$; see figure 2. To avoid overcounting configurations, we identify the height $h$ with $h+4$. The flip operator

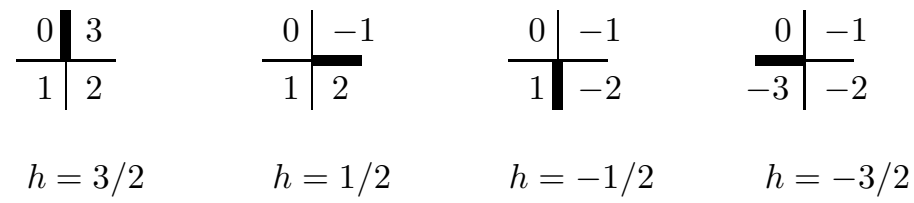

FIG. 2: 4 height configurations

$\hat{F}$ corresponds to changing $h \rightarrow h \pm 1$ on all four sites around a plaquette (the \pm depending on the sub-lattice). Columnar order for dimers corresponds to an expectation value for $h$, while staggered order for dimers corresponds to an expectation value for $\partial h$. One can obtain a Hamiltonian with ordered ground states by allowing the coefficients of the two terms in Eq. (2.2) to be different. If the coefficient of $\hat{F}_{i}$ is larger, this favors columnar order; if the coefficient of $\hat{V}_{i}$ is larger, staggered order is favored. It is widely assumed that $H_{R K}$, where the coefficients are equal, describes a phase transition between the two kinds of order [12,23 70,78$]$. However, this has never been proven, and there exists the possibility of an intermediate "plaquette" phase [79].

We would now like to take the continuum limit of the quantum dimer model in its height description. In this limit, we identify the height $h$ with a scalar field $4 \varphi(x)$. Like the height, the scalar field must be periodic, so we identify $\varphi$ with $\varphi+1$. We have already noted that the continuum correlators of the square-lattice quantum dimer model are those of a massless Dirac fermion. The correlators in the ground state of the quantum Hamiltonian in terms of $\varphi$ must be identical. The quantum Lifshitz Hamiltonian Eq. (2.4) and Eq. (2.9) has ground-state correlators of the form given in Eq. (2.14). When $\kappa^{-1}=2 \pi$, these correlators are precisely those of a Dirac fermion; this is a result of the widely-known procedure known as bosonization [48]. In fact, correlators of many two-dimensional critical classical statistical mechanical systems, not just free fermions, can be written in terms of exponentials of a free boson [69]; we collect some of these results in Appendix A. In the next section we will display quantum lattice models whose continuum limit corresponds to all values of $\kappa$.

The Hamiltonian $H_{R K}$ on the square lattice in the continuum limit is therefore identified with the quantum Lifshitz Hamiltonian with $\kappa^{-1}=2 \pi$. This also allows a qualitative understanding of the physics away from the RK point [53]. A phase with staggered order should have an expectation value of $\partial_{x} \varphi$ or $\partial_{y} \varphi$ in the continuum theory. Adding a term $(\nabla \varphi)^{2}$ to the Hamiltonian with negative coefficient will drive the system into such an ordered phase. Adding this term with positive coefficient will favor a constant value of $\varphi$, driving the system into columnar order. Adding terms like $\cos (2 \pi \varphi)$ will also drive the system into a phase with columnar order. Thus one expects that at a critical point like that described by $H_{R K}$ on the square lattice, the coefficients of $(\nabla \varphi)^{2}$ and $\cos (2 n \pi \varphi)$ will

\footnotetext{
4 This construction has been employed extensively in statistical mechanics models of classical dimers and loops; see for instance [7].
} 
vanish. This leaves Eq. (2.4) as the simplest non-trivial Hamiltonian with the desired properties. The requirement that the ground state be equivalent to a free fermion then fixes the coefficient $\kappa$; note that if desired $\kappa$ can scaled out of the Hamiltonian by redefining the compactification relation to be $\varphi \sim \varphi+\sqrt{\kappa}$.

It is not at all clear whether critical $2+1$-dimensional field theory and the continuum limit of $H_{R K}$ are identical for excited states, although the above heuristic argument is very suggestive. The Hamiltonian for the scalar field theory Eq. (2.9) is purely quadratic in the field $\varphi$, so one can obtain essentially any desired information exactly. $H_{R K}$ is not so simple on the lattice, but one may hope the two models are in the same universality class. In any event, we can easily extract all the excited-state energies for the quantum Lifshitz field theory. The operators $Q(x)$ and $Q^{\dagger}(x)$ are essentially harmonicoscillator creation and annihilation operators: the equal-time commutation relation Eq. (2.6) implies that

$$
\left[Q(\vec{x}), Q^{\dagger}(\vec{y})\right]=\kappa \nabla^{2} \delta^{(2)}(\vec{x}-\vec{y})
$$

The ground state is indeed annihilated by all $Q(x)$, so we can create excited states by acting with $Q^{\dagger}(x)$. The commutation relations Eq. (3.5) mean that the dispersion relation is $E=\kappa p^{2}$. This theory is gapless but not Lorentz-invariant: the dynamical critical exponent is $z=2$.

The exponent $z=2$ can also be seen by looking at the classical action associated with this Hamiltonian. This will also allow us to make contact with the earlier three-dimensional statistical-mechanical results. The action consistent with the Hamiltonian of Eq. (2.4) and the canonical commutation relations of Eq. (2.6) is

$$
\mathcal{S}=\int d^{3} x\left[\frac{1}{2}\left(\partial_{t} \varphi\right)^{2}-\frac{\kappa^{2}}{2}\left(\nabla^{2} \varphi\right)^{2}\right]
$$

Clearly, this action is not Lorentz invariant and has $z=2$. It is rotationally invariant only in the XY plane. Defining the imaginary time $\tau=i t$ gives the Euclidean action (2.5). The imaginary time axis $\tau$ can be regarded as the $z$-coordinate of a three-dimensional classical system in which $\varphi(\vec{x}, \tau)$ is an angle-like variable and the action represents the spin-wave approximation of an anisotropic classical $X Y$ model. In general one would have expected a term proportional to the operator $(\nabla \varphi)^{2}$ with a finite positive stiffness in the plane. This is so in the $X Y$ ferromagnetic phase. On the other hand, if the stiffness becomes negative, there is an instability to a modulated helical phase. The action of Eq. (2.5) represents the Lifshitz point, the critical point of this phase transition [54]80] where the stiffness vanishes. This effective action also plays a central role in the smectic A-C transition [81] and in other classical liquid crystal phase transitions associated with the spontaneous partial breaking of translation and/or rotational invariance [2,82]. The compactified version of the problem (i.e., the identification $\varphi \sim \varphi+1$ ) has also been considered in this context, for example in Ref. [54]. The choice of period in general depends on the physical context of the problem.

The square-lattice quantum dimer model and the scalar field theory with Hamiltonian Eq. (2.9) are both at critical points, in that the correlators in the ground state are algebraically decaying. The quantum dynamics implied by this Hamiltonian must be compatible with the 2D time-independent conformal invariance. In particular, the spectrum of the quantum theory must be gapless and, as we learned from this example, the dynamic critical exponent must be $z=2$. Notice however, that $z=2$ alone does not guarantee a gapless (or even critical) theory. Indeed, instructive counter-examples to this statement are well known in the theory of (the absence of) quantum roughening [83,84] where quantum fluctuations destroy the critical behavior and lead to an ordered state through an order-fromdisorder mechanism. 


\section{The off-critical field theory}

We have thus shown that the continuum limit of the square-lattice quantum dimer model is described by the quantum Lifshitz model at a special point $\kappa^{-1}=2 \pi$. We also argued that at least some deformations of the two models result in ordered phases. However, we saw at the beginning of this section that not all deformations of the square-lattice quantum dimer model result in an ordered phase. Allowing dimers across the diagonals with Hamiltonian $H_{w}$ results in a topologically-ordered phase, where the order parameter is not local.

In this subsection, we find a bosonic field theory describing the topological phase in the continuum limit. We showed above that in the scaling limit $w \rightarrow 0$ with $w / a$ finite, the ground-state wave function Eq. (3.4) can be written in terms of a free massive Dirac fermion of mass proportional to $w / a$. In two dimensions, the bosonic version of a massive Dirac fermion is the sine-Gordon model at a particular coupling. Precisely, the two-dimensional fermion action is equivalent to

$$
S_{2 d}=\int d^{2} x\left[\kappa(\nabla \varphi)^{2}-\lambda \cos (2 \pi \varphi)\right]
$$

For the free-fermion case, we have $\kappa=1 / 2 \pi$; we will discuss the more general case in section IV. In the fermion language, different values of $\kappa$ correspond to adding a four-fermion coupling to $S_{2 d}$. To find a Hamiltonian with this two-dimensional action describing the ground state, we again find an operator $Q(x)$ and define the Hamiltonian via Eq. (2.9). The operator

$$
Q(x) \equiv \frac{1}{\sqrt{2}}\left(\frac{\delta}{\delta \varphi}+\kappa \nabla^{2} \varphi+\frac{\lambda}{2 \pi} \sin (2 \pi \varphi)\right)
$$

annihilates the wave functional $\Psi \propto e^{-S_{2 d}}$. Because of the extra term in $Q$, the commutator $\left[Q(\vec{x}), Q^{\dagger}(\vec{y})\right]$ is not a simple c-number, but in fact depends on the field configuration $\varphi$,

$$
\left[Q(\vec{x}), Q^{\dagger}(\vec{y})\right]=\kappa \nabla^{2}\left(\delta^{(2)}(\vec{x}-\vec{y})\right)+\lambda \sin (2 \pi \varphi(\vec{x})) \delta^{(2)}(\vec{x}-\vec{y}) .
$$

Thus, normal-ordering the Hamiltonian is not just an innocent ground state energy shift: the two parts of (2.9) are not the same here. To obtain the desired ground-state wave functional, we must define $H$ of the form $\int Q^{\dagger} Q$. The three-dimensional version of this model was discussed in Ref. [54].

This Hamiltonian is not quadratic in the field $\varphi$ except at the critical point $m=0$, so that even with this fine tuning this model cannot be solved simply. Since $Q$ and $Q^{\dagger}$ do not have simple commutation relations, we cannot simply find the spectrum of this theory. Of course, one can compute properties in the fermionic picture, but as noted before, computations involving spin fields are non-trivial in this basis as well. However, since there are dimensionful parameters in the Hamiltonian and no spontaneous breaking of a continuous symmetry, it seems likely that the Hamiltonian is gapped. Moreover, in the limit with $\lambda / \kappa$ finite, the action Eq. (3.7) reduces to that of a free massive boson. Then one can solve the model explicitly, and the quantum Hamiltonian indeed has a gap. When $\lambda$ is reduced to a finite value, the gap should remain ${ }^{5}$.

\footnotetext{
5 The equal-time correlators of the vertex operators defined and computed in Appendix $\mathrm{A}$ can also be computed away from the critical point by either by a naive semi-classical argument, which predicts the simple exponential decay we just discussed, or in a more sophisticated way by means of form factors [68]. Apart from a subtle bound state structure in the spectral functions, the more sophisticated approach confirms the essence of the naive semi-classical result. On the other hand, the $z=2$ character of the critical theory suggests that time-dependent correlation functions must be consistent with this fact, and that the correlators must be functions of $x^{2}$ or $t$, and that the time dependent Euclidean auto-correlation functions may obtained from the equal-time correlator by replacing $x^{2} \leftrightarrow|t|$. These arguments are consistent with the renormalization-group results of Ref. [54]
} 
There are many terms in the Hamiltonian of this field theory, and their coefficients must be finetuned to enable us to compute the ground-state wave function explicitly. There are terms like $\cos (4 \pi \varphi)$ and $(\nabla \varphi)^{2}$ which, as noted above, tend to order the system. However, the exact lattice results for $H_{w}$ from [11] show that the ground-state correlators for $w$ small are those of the bosonic Hamiltonian with these special couplings. Thus this model is not ordered but rather topologically ordered. Moreover, since the model is gapped, we expect that its physical properties are robust and persist even when the coefficients are tuned away from this special point. Thus there must exist a topological phase, not just an isolated point. An interesting open problem is to understand how large (in coupling constant space) the topological phase is, as compared to the ordered phases.

\section{A QUANTUM EIGHT-VERTEX MODEL}

In the last section, we discussed a $2+1$-dimensional theory whose ground-state wave function is simply described in terms of a classical two-dimensional bosonic field. With vanishing potential and a particular value of the coupling $\kappa$, it is believed to describe the continuum limit of the quantum dimer model on the square lattice. In this section, we will study lattice models which in the continuum limit allow arbitrary values of $\kappa$. These models also have a quantum critical line separating an ordered phase from a topologically-ordered phase.

The degrees of freedom in our model are those of the classical two-dimensional eight-vertex model. These are arrows placed on the links of a square lattice, with the restriction that the number of arrows pointing in at each vertex is even. This means that there are eight possible configurations at each vertex, which we display in figure (3). The classical Boltzmann weights for a given vertex in the

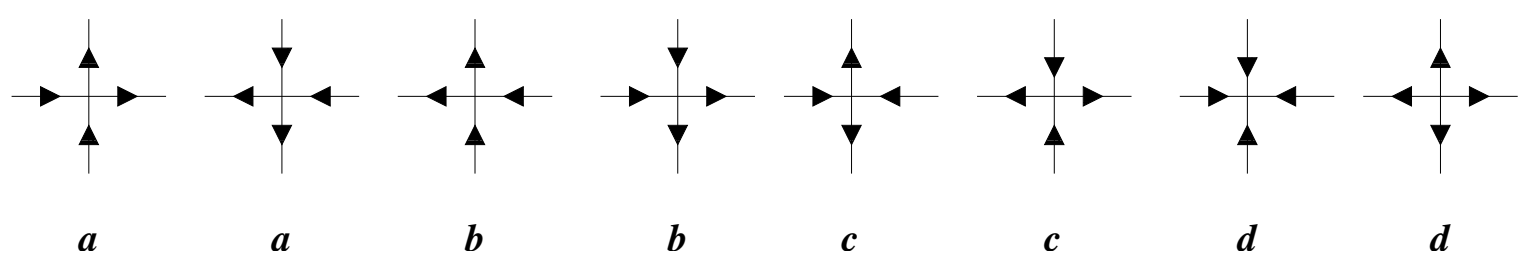

FIG. 3: The eight vertices and their Boltzmann weights

zero-field eight-vertex model are usually denoted by $a, b, c$ and $d$, as shown in the figure. Since we are interested in rotationally-invariant theories, we set $a=b$ in the following; moreover, since we can rescale all the weights by a constant, we set $a=b=1$. A typical configuration is displayed in figure (4); the Boltzmann weight of such a configuration is given by the product of Boltzmann weights of the vertices.

The classical eight-vertex model is integrable, and many of its properties can be derived exactly [85]. For $a=b=1$, it has ordered phases for $c>d+2$ and $d>c+2$. In these phases the $\mathbb{Z}_{2}$ symmetry of flipping all the arrows is spontaneously broken. Critical lines with continuously varying exponents at $c=d+2$ and $d=c+2$ separate the ordered phases from the disordered one $|c-d|<2$. The correlation length diverges as 85$]$

$$
\xi \sim|| c-d|-2|^{-\pi /(2 \mu)}, \quad \mu \equiv 2 \tan ^{-1}(\sqrt{c d}),
$$

near these critical lines. (For $\pi / \mu$ an even integer this is multiplied by $\log || c-d|-2|$.) When $c=0, d \leq 2$ or $d=0, c \leq 2$, the model is also critical; in fact the partition function on this line can be mapped onto that for the order-disorder critical line. For $d=0$, the exponent in Eq. (4.1) diverges: there is a Kosterlitz-Thouless transition as one brings $c$ through 2. Another useful result for the classical correlation length is that it is zero on the line $c=d$; this is the state of maximal disorder. 


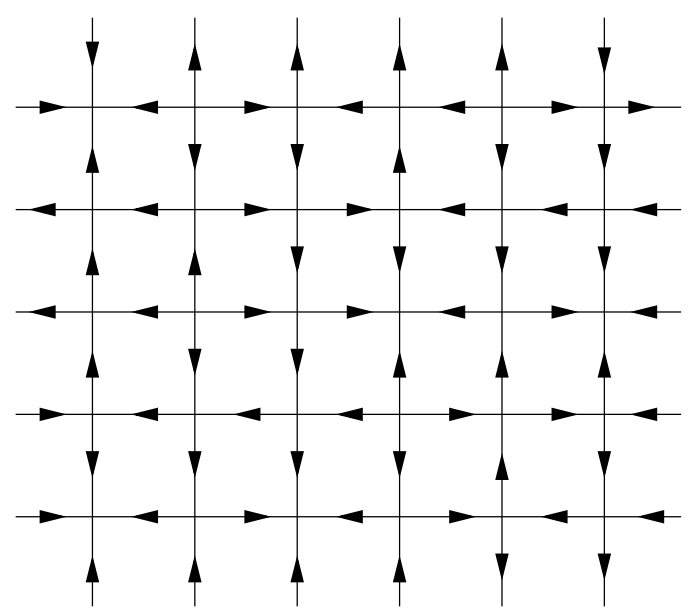

FIG. 4: A typical configuration in the eight-vertex model

An order parameter which will be useful later comes by rewriting the model in terms of an Ising spin at the center of each plaquette. This description is best thought of as two Ising models, with spins $\tau(A)$ on one sublattice, and $\tau(B)$ on the other. Then the Boltzmann weights can be written in terms of two Ising couplings between nearest sites on the $A$ lattice and on the $B$ lattice, and a four-spin coupling between the two $A$ and two $B$ spins around a site of the original lattice. The polarization operator of the eight-vertex model becomes $\tau(A) \tau(B)$. One finds that its expectation value is non-vanishing in the ordered phase, and vanishes in the disordered phase $|c-d|<2$ [85]. One also can define Neél-like staggered order parameters, in terms of $\tau(A)$ and $\tau(B)$ individually, which do not vanish in the ordered phase. Along the line $c d=1$, the four-spin coupling vanishes, so the eight-vertex model turns into two decoupled Ising models; the model here in this case be solved by using Pfaffian techniques [86]. Along the line $c=d$, the two Ising couplings vanish, leaving only the four-spin coupling. Thus this line in the classical model has an extra $\mathbb{Z}_{2}$ gauge symmetry.

The classical eight-vertex model has a number of useful dualities 85]. They can be described by defining the combinations $W_{1}=(a+b) / 2, W_{2}=(a-b) / 2, W_{3}=(c+d) / 2$ and $W_{4}=(c-d) / 2$. The partition function is invariant under the exchange of any two of the $W_{j}$ and under the $W_{j} \rightarrow-W_{j}$ for any $j$. These dualities, for example, map the critical line $c=d+2$ to the critical line $d=0, c \leq 2$ by exchanging $W_{1}$ with $W_{4}$. In Ising language this amounts to performing Kramers-Wannier duality on one of the two types of Ising spins. The line $c=d+2$ is invariant under the exchange $W_{1} \leftrightarrow W_{3}$. In Ising language, this duality amounts to taking the Kramers-Wannier dual of both types of Ising spins. Denoting the dual spins as $\mu(A)$ and $\mu(B)$, duality means therefore that in the disordered phases, the expectation value $\langle\tau(A) \tau(B)\rangle$ is non-vanishing.

\section{A. Construction of the quantum eight-vertex Hamiltonian}

We now define a quantum Hamiltonian acting on a Hilbert space whose basis elements are the states of this classical eight-vertex model. To define such a Hamiltonian, we first need the analog of the flip operator in the quantum dimer model. A flip operator needs to be ergodic: by flips on various plaquettes one should be able to reach all the states with the same global conserved quantities. The simplest such operator for the eight-vertex model is the operator which reverses all the arrows around a given plaquette. We write this flip operator $\hat{\mathcal{F}}_{i}$ explicitly in gauge-theory language in appendix C] Note that as opposed to the quantum dimer model on the square or triangular lattice, all configurations 
in the quantum eight-vertex model are flippable ${ }^{6}: \hat{\mathcal{F}}_{i}$ preserves the restriction that an even number of arrows be pointing in or out at each vertex.

The simplest Hamiltonian has no potential energy, just a flip term. It is convenient to write this in terms of a projection operator: for $I$ the identity matrix, we have $\left(I-\hat{\mathcal{F}}_{i}\right)^{2}=2\left(I-\hat{\mathcal{F}}_{i}\right)$. Then the Hamiltonian

$$
H_{c=d=1}=\sum_{i}\left(I-\hat{\mathcal{F}}_{i}\right)
$$

has a ground state corresponding to the equal-amplitude sum over all eight-vertex model states. In terms of the Boltzmann weights introduced above, this is the state with $a=b=c=d=1$. A Hamiltonian with the same ground state was introduced by Kitaev [42]. There the eight-vertex-model restriction of having an even number of arrows in and out at each vertex was not required a priori, but instead a term was introduced giving a positive energy to vertices not obeying the restriction. The zero-energy ground state can therefore include no such vertices, so the ground state for the model of [42] is indeed the sum over the eight-vertex-model configurations with equal weights. Because every plaquette is flippable and all configurations have equal weights, different $\hat{\mathcal{F}}_{i}$ commute. Therefore all the terms in Eq. (4.2) commute with each other, so they can be simultaneously diagonalized and their eigenstates can easily be found, as for the quantum Lifshitz field theory. The model is gapped, and is in a topologically-ordered phase [42]. This follows as well from the results for the classical eight-vertex model discussed above: for $c=d=1$ all Ising couplings vanish resulting in two decoupled Ising models at infinite temperature. At this point the order parameter vanishes, but the non-local order parameter does not. We can thus interpret the product of dual Ising variables $\mu(A) \mu(B)$ as a topological order parameter.

This ground state can also be mapped onto the ground state of a $\mathbb{Z}_{2}$ gauge theory deep in its deconfined phase. In Appendix $\mathbb{C}$, we give a detailed derivation of the $\mathbb{Z}_{2}$ gauge theory of the full quantum eight-vertex model we define below, as well as the its dual theory which we will use to characterize some of the phases. In Appendix $\mathbb{B}$ we discuss the $U(1)$ gauge theory description of the quantum six-vertex model, which describes the limit $d=0$, and by duality, the lines $c=0, c^{2}=d^{2}+2$ and $d^{2}=c^{2}+2$.

We now find a two-parameter quantum Hamiltonian whose ground state is a sum over the states of the eight-vertex model with amplitudes given by the classical Boltzmann weights with arbitrary $c$ and $d$. We are still keeping $a=b=1$ to preserve two-dimensional rotational invariance, but this restriction can be relaxed if desired. Our model is neither the simplest nor the most natural extension of the classical eight-vertex model: a simpler Hamiltonian was proposed by Chakravarty [89] in the context of $d$-density waves. Although we believe that our Hamiltonian and Chakravarty's describe the same physics, we are not aware of any simple mapping between these models. Another lattice model related to the quantum eight-vertex model discussed here was introduced in Ref. [90]. The main virtue of the construction that we use here is the structure of the ground-state wave function.

Finding a quantum Hamiltonian with a known ground state is straightforward to do by using the trick discussed above (and in Ref. [58]). Namely, we find a Hamiltonian of the form

$$
H_{q 8 v}=\sum_{i} w_{i} \mathcal{Q}_{i}
$$

where $\mathcal{Q}_{i}=\mathcal{Q}_{i}^{\dagger} \propto \mathcal{Q}_{i}^{2}$. To yield the desired ground state, each operator $\mathcal{Q}_{i}$ must annihilate the sum over states with each state weighted by $c^{N_{c}} d^{N_{d}}$, where $N_{c}$ and $N_{d}$ are the number of $c$ and $d$ type

\footnotetext{
${ }^{6}$ However, the quantum dimer models on the Kagome [87] and Fisher [88] lattices have Hamiltonians where all plaquettes are flippable.
} 
vertices in that state. In particular, we look for a $\mathcal{Q}_{i}$ of the form

$$
\mathcal{Q}_{i}=\sum_{i}\left[\hat{\mathcal{V}}_{i}-\hat{\mathcal{F}}_{i}\right]
$$

where $\hat{\mathcal{V}}_{i}$ is diagonal and depends on the Boltzmann weights for the four vertices at the corners of the plaquette $i$. Since $\left(\hat{\mathcal{F}}_{i}\right)^{2}=I$, this Hamiltonian breaks into 2 by 2 blocks like $H_{R K}$ for the quantum dimer model. If we choose the potential $\mathcal{V}_{i}$ so that the blocks are of the form

$$
\left(\begin{array}{cc}
v & -1 \\
-1 & v^{-1}
\end{array}\right)
$$

$\mathcal{Q}_{i}$ will have the desired properties.

To find $\hat{\mathcal{V}}_{i}$, let $n_{c}$ be the number of $c$-vertices at the corners of the plaquette $i$, and let $\widetilde{n}_{c}$ be the number of $c$-vertices around the plaquette after it is flipped by $\hat{\mathcal{F}}_{i}$. Likewise, let $n_{d}$ be the number of $d$-vertices around the plaquette, while $\widetilde{n}_{d}$ is the number of $d$ vertices in the flipped configuration. Note that $\hat{\mathcal{F}}_{i}$ always flips a $c$ or $d$ vertex to an $a$ or $b$ vertex, and vice versa. Consequently, we take the operators $\hat{\mathcal{V}}_{i}$ to be of the form

$$
\hat{\mathcal{V}}_{i}=c^{\widetilde{n}_{c}-n_{c}} d^{\widetilde{n}_{d}-n_{d}}
$$

Explicitly, we can write the projectors as follows

$$
\mathcal{Q}_{i}=\left(\begin{array}{cc}
c^{\widetilde{n}_{c}-n_{c}} d^{\widetilde{n}_{d}-n_{d}} & -1 \\
-1 & c^{\widetilde{n}_{c}-n_{c}} d^{\widetilde{n}_{d}-n_{d}}
\end{array}\right) .
$$

This $\mathcal{Q}_{i}$ is indeed proportional to a projection operator: the Hamiltonian (4.3) has the classical eightvertex model as an eigenstate. This holds for any choice of the $w_{i}$, but in the $\mathbb{Z}_{2}$ gauge-theory language of appendix [C it is natural to set all $w_{i}=1$. The amplitude of the ground-state wave function of this $H_{q 8 v}$ on a state with $N_{c} c$-vertices and $N_{d} d$-vertices is

$$
\Psi_{0}\left[N_{c}, N_{d}\right]=\frac{c^{N_{c}} d^{N_{d}}}{\sqrt{Z\left(c^{2}, d^{2}\right)}},
$$

where $Z(c, d)$ is the partition function of the classical two-dimensional eight-vertex model with weights $a=1, b=1, c$ and $d$. The arguments in the denominator are $c^{2}$ and $d^{2}$ because averages in the quantum model are calculated with respect to $\left|\Psi_{0}\right|^{2}$.

In section III we discussed how by taking the limit $w \rightarrow 0$ in $H_{w}$, one can recover a slightlygeneralized square-lattice quantum dimer model which allows defects with no dynamics. Similarly, here one can find a quantum six-vertex model with some defects allowed by taking the limit $d \rightarrow$ $0 .{ }^{7}$ Configurations with $n_{d}>\widetilde{n}_{d}$ on any plaquette will receive infinite potential energy and so are disallowed. Some useful facts are that $n_{c}+n_{d}+\widetilde{n}_{c}+\widetilde{n}_{d}=4$, and $n_{c}-\widetilde{n}_{c}=0 \bmod 2$ and $n_{d}-\widetilde{n}_{d}=0$ $\bmod 2$. When $d \rightarrow 0$, configurations with $n_{d}=\widetilde{n}_{d}=0$ are the flippable plaquettes of the six-vertex model, and are obviously included in the ground-state wave function. Configurations with $n_{d}=\widetilde{n}_{d}=1$ or $n_{d}=\widetilde{n}_{d}=2$ are not suppressed, but the flip therefore preserves the number of $d$ vertices around each plaquette. Thus in the $d \rightarrow 0$ limit we can view these $d$ vertices as defects in the quantum six-vertex model. The ground-state wave function is a sum over all allowed states with a given set of plaquettes with defects. The amplitude of each configuration in this sum is proportional to $c^{N_{c}}$. We discuss the relation between the quantum six- and eight-vertex models in more detail in the Appendices $\mathbb{B}$ and $\mathbb{C}$.

\footnotetext{
7 This Hamiltonian in the special case $c=1, d=0$ was discussed in Ref. 91]. Moreover, the quantum six-vertex model should be in the same universality class as the "supersymmetric" $X Y$ model introduced some time ago 92]; this model defines a similar Hamiltonian acting on the 2D classical XY model, and presumably can also be understood in terms of a mapping to the quantum Lifshitz model. Another quantum six-vertex model has been proposed as a model of a planar pyrochlore lattice in Ref. [93]. This six-vertex model is not of the Rokhsar-Kivelson type; it is the six-vertex limit of the simpler quantum eight-vertex model we discuss in section IVD
} 


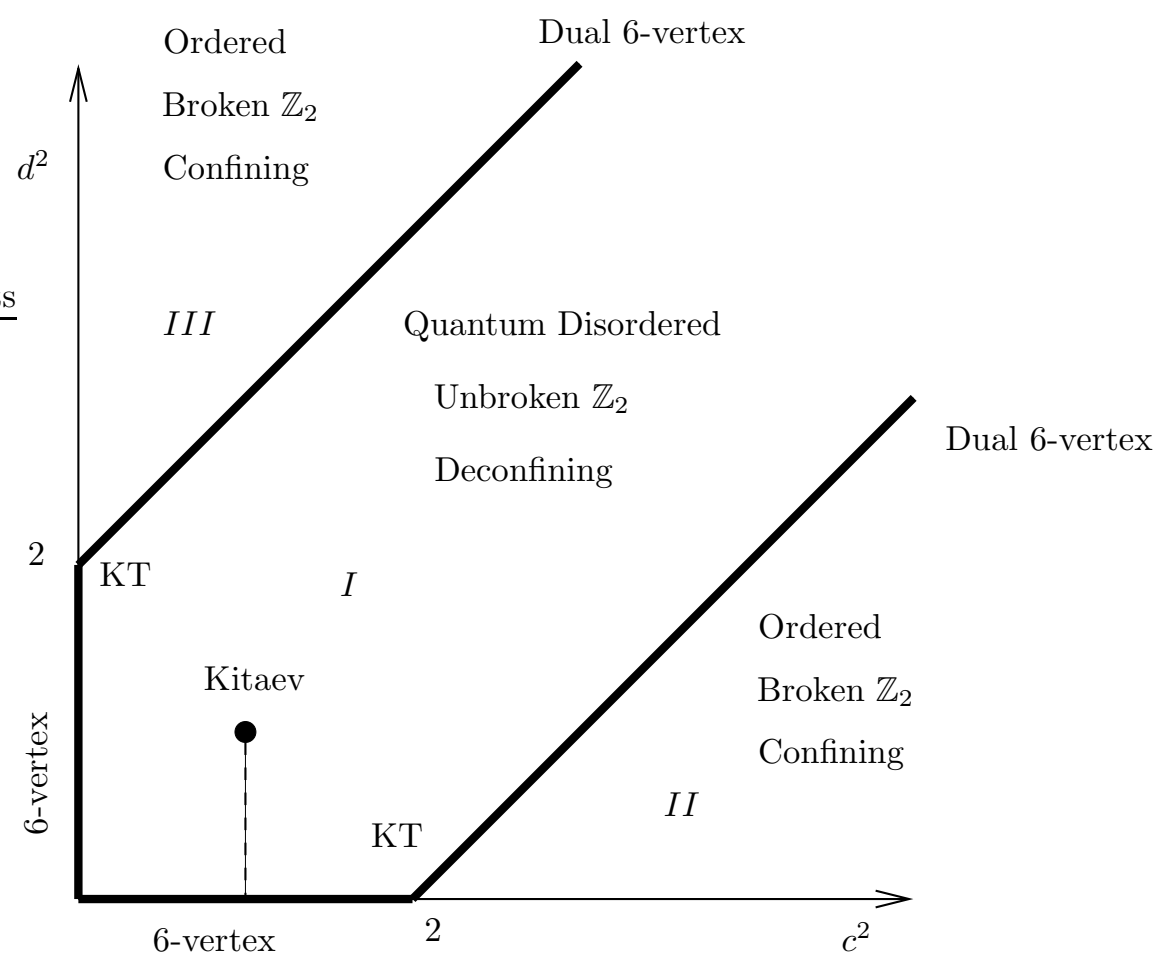

FIG. 5: Phase diagram of the quantum eight-vertex model: phases $I$ and $I I$ are separated by a dual 6-vertex transition (same with $I$ and $I I I$ ); 6 -vertex denotes the 6 -vertex model critical lines and KT are $2 D$ KosterlitzThouless transitions; the dotted line shows that the Kitaev point is smoothly connected to the critical regime of the eight-vertex model.

\section{B. The phase diagram}

Now that we have the exact ground-state wave function for the quantum eight-vertex Hamiltonian of (4.3), we can use it to determine the phase diagram. Given the fact that the probability of a configuration of arrows in the ground state wave function is equal to the Boltzmann weight of a classical eight-vertex model, we can deduce much of the physics of the quantum theory (at least its equal-time properties) directly from the Baxter solution of the classical 2D eight-vertex model [85], as well as from Kadanoff's classic work on its critical behavior 69 94 95]. The only change is that the weights must be squared here, since in quantum mechanics we weigh configurations with $|\Psi|^{2}$. The phase diagram is displayed in figure 5 note that the axes are labeled by $c^{2}$ and $d^{2}$.

We will now use this knowledge, as well a simple perturbative arguments in the quantum theory, to determine the phase diagram, the behavior of physical observables in the different phases, and (much of) their critical behavior. A useful fact is that for $c d=1$, the classical model with partition function $Z\left(c^{2}, 1 / c^{2}\right)$ is equivalent to two decoupled Ising models. This decoupling is a property only of the wave function, not of the Hamiltonian of the full $2+1$-dimensional quantum theory. Since this line $c d=1$ goes through both ordered and disordered phases, much of the physics of the quantum eight-vertex model can be described at least qualitatively in terms of decoupled Ising models. In particular, for any values of $c$ and $d$ except those on the critical line, correlators decay exponentially fast with distance with a correlation length $\xi$ which diverges as the phase boundary is approached, in a manner given in Eq. (4.1). This exponential decay occurs in general, not just on the decoupling curve.

As with the dimer models discussed in section III the partition function for $c d=1$ can be expressed in terms of Grassmann variables with only quadratic terms, i.e. free fermions. Duality means that 


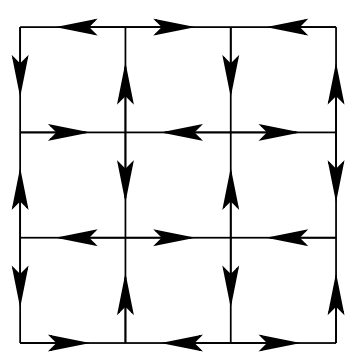

FIG. 6: The ordered phase "antiferroelectric" or "staggered flux" phase of the eight-vertex model for $c \gg 1$. In this limit there are only $c$ vertices in the ground state.

$Z\left(c^{2}, d^{2}\right)$ is free-fermionic for $c^{4}+d^{4}=2$ as well. The correlators in the continuum limit of the (critical) square-lattice quantum dimer model are therefore identical to those obtained for $c=\sqrt{2}$, $d=0$. The special point $c=d=1$ discussed above and in Ref. [42] (labeled in fig. [5 as "Kitaev") is also free fermionic. At this point one does not need the Pfaffian techniques to compute correlators exactly, and one finds that the model is in a disordered phase in the Ising-spin language. However, the expectation value $\langle\mu(A) \mu(B)\rangle$ is non-vanishing, so there is topological order at this point.

Let us now discuss the different phases of this system.

1. The Ordered (Confined) Phase:

From the known phase diagram of the classical eight-vertex model [85], we conclude that the ground state of the quantum model with the Hamiltonian of Eq. (4.3) has an ordered phase for $c^{2}>d^{2}+2$ (and also for $d^{2}>c^{2}+2$ ). That this is an ordered phase can be seen easily by considering the limit $c \rightarrow \infty$ (with $d$ fixed). In this limit the ground state is dominated by just two configurations, related to each other by a lattice translation of one lattice spacing, which have a $c$ vertex on every site, as shown in fig. [6] In this phase the staggered polarization operator $\langle\tau(A) \tau(B)\rangle$ has a non-vanishing expectation value. This result can also be obtained directly from the Hamiltonian of the $\mathbb{Z}_{2}$ gauge theory, Eq. (4.11), since for $c$ large the potential energy term $H_{V}$ dominates and in it the piece associated with the $c$ projection operators.

The ordered phase is also confining. Below we will discuss the behavior of the Wilson loop operator and show that in this regime it obeys an area law, the hallmark of confinement [31,57]. We will also show that the energy of a state with two static sources grows linearly with their separation. We should also note here that the equal-time fermion correlation function has an exponential decay in this phase, suggesting that this phase may support massive fermionic excitations.

2. The Disordered (Deconfined) Phase:

From the exact solution of the classical model, we know that there is a disordered phase for $c^{2}<d^{2}+2\left(d^{2}<c^{2}+2\right)$ (see Fig. [5). This is also a deconfined phase. This is most easily seen by taking a point deep in this phase, such as the Kitaev point $a=b=c=d=1$ [42]. In fact, all along the line $a=b=1$ and $c=d$, the correlation length of the eight-vertex model is zero [85]. All points in the disordered phase have this line as their RG stable fixed point (all points on the line are equivalent). This is the infinite temperature limit of the classical 2D eight-vertex model. In this regime the expectation value of the polarization operator $\langle\tau(A) \tau(B)\rangle$ is zero (and its correlation vanishes at a length scale of the lattice spacing.). Thus this state does not exhibit long-range order, but since the dual variable $\langle\mu(A) \mu(B)\rangle$ is non-vanishing, it exhibits topological order. Quantum mechanically, the wave function is the equal-amplitude superposition of all configurations of arrows consistent with the eight-vertex restrictions. This state is deconfined since in this limit the Hamiltonian reduces to the flip term (plaquette). It 
is well known 31] that in this regime the Wilson loop operator has a perimeter law behavior and that the energy of two static sources is finite and independent of their separation. The low energy ( $i$. e. long-distance) sector of this phase describes a topological $\mathbb{Z}_{2}$ deconfined theory, equivalent to the Kitaev point.

\section{Critical Behavior:}

The $2 \mathrm{D}$ quantum theory has lines of critical points $c^{2}=d^{2}+2$ (and $\left.d^{2}=c^{2}+2\right)$. There are also lines of critical points for $d=0$ and $0 \leq c^{2} \leq 2$ (and $c=0$ and $0 \leq d^{2} \leq 2$ ). All of these critical lines are (up to duality transformations) equivalent to the six-vertex model. Notice that, since the energy of this quantum state is exactly zero (by construction), the ground-state energy does not have singularities at the phase transitions. This is of course a peculiarity (or rather a pathology) of this model and it certainly non-generic: any perturbation leading to a non-vanishing energy will lead to singularities in the energy of the ground state.

In section [IIB, we discussed how the ground-state correlators of the (multi) critical square-lattice quantum dimer model were the same as those of the quantum Lifshitz theory, Eq. (2.4), at $\kappa^{-1}=2 \pi$ 14,53]. The same equivalence holds for the quantum eight-vertex model for all values of $\kappa$. Moving along the critical line results in changing $\kappa$ as given in (4.94.10). Kadanoff 69, 94, 95] showed that the critical behavior of the classical eight-vertex model can be mapped exactly to the critical behavior of the two-dimensional Gaussian model, the free boson described by Eq. (2.12) and Eq. (2.13). The equal-time correlators in the quantum Lifshitz model summarized in Appendix $\AA$ are therefore identical to those of the quantum eight-vertex model on its critical lines. Moreover, when $d=0$ and $c^{2} \leq 2$, the eight-vertex model becomes the six-vertex model, which has a height description. Thus the same heuristic arguments applied in the last section can be applied here, again implying that the quantum six-vertex model and the quantum Lifshitz theory are in the same universality class. In particular, the dynamics of the quantum eight-vertex model along its critical lines will obey scaling with a dynamic exponent $z=2$.

To compete the identification, we need to express the coupling constant $\kappa$ of the quantum Lifshitz model in terms of the eight-vertex parameters $c$ and $d$. This is easiest to do by computing the dimension $x$ of the "energy" operator, which when added to the action of (classical) critical theory, moves it away from criticality. Combining universality with Baxter's exact result (4.1) for the correlation-length exponent gives $x=2(1-\mu / \pi)$. We have normalized the boson $\varphi$ in Appendix A so that the energy operator is given by $\cos (2 \varphi)$, which has dimension $x=1 /(2 \pi \kappa)$. Note that at the free-fermion point $\kappa^{-1}=2 \pi$, the energy operator has dimension 1 , the dimension of a $2 \mathrm{D}$ fermion mass term. Combining the two and using a simple trigonometric identity yields

$$
\kappa^{-1}=8 \cot ^{-1}(c d) . \quad \text { for }\left|c^{2}-d^{2}\right|=2
$$

and by duality

$$
\kappa^{-1}=8 \cot ^{-1}\left(\sqrt{\frac{4}{c^{4}}-1}\right) \quad \text { for } c^{2} \leq 2, d=0 .
$$

At the Kosterlitz-Thouless transition point $c^{2}=2, d=0$ where the two critical lines meet, the dimension $x=0$ as expected, and both formulas give $\kappa^{-1}=4 \pi$ here.

This equivalence to a scalar field theory can be extended to the scaling region near the critical lines, by repeating the arguments of section IIIC. The perturbing operator is $\cos (2 \varphi)$; symmetry forbids $\cos (\varphi)$ from being added to the action of the classical theory. Thus near to the critical lines the effective field theory will be of the form (3.8) and Eq. (2.9); these field theories will have the correct critical exponents. 


\section{Operators}

Equal-time correlation functions of operators which are diagonal in the arrow representation of the eight-vertex model are identical to correlation functions of the classical eight-vertex model. Unfortunately, these correlation functions can be computed exactly only along the free-fermion lines, by using Pfaffian techniques. Likewise correlation functions of off-diagonal operators (e.g. flip operators) can only be determined at some special points deep in the ordered and disordered phases by using directly the quantum eight-vertex Hamiltonian. However, at or near the critical lines, the asymptotic behavior of the correlators can be found by using universality. The operators of interest in the eight-vertex model can all be expressed in terms of the charge and vortex operators of the Gaussian model [95].

The operators of interest in the quantum eight-vertex model can be readily identified in the quantum Lifshitz model. Thus we have a full identification of a $2+1$-dimensional quantum critical theory. As a direct consequence of being able to identify these operators we can also study how these operators perturb the critical theory, at least within a renormalization group argument along the lines of Ref. 54]; the analog in the quantum dimer model is our analysis of $H_{w}$ for $w$ small at the end of section 2.

To identify the eight-vertex operators in the bosonic language as in Ref. [95], we first study the six-vertex line, where there is a height description. There the height is local in terms of spin variables, so we expect the boson $\varphi$ to be local as well. Standard bosonization techniques [96] show that only products of spin operators from both Ising models can be written in terms of the boson. One finds that $\cos (\varphi) \sim \tau(A) \tau(B)$. Note that this is consistent with the identification of $\cos (2 \varphi)=2 \cos ^{2}(\varphi)-1$ with the perturbing operator; the usual Ising operator product gives the fusion rule $\tau(A) \tau(A) \sim 1+\mathcal{E}(A)$, where $\mathcal{E}(A)$ is the energy operator.

To study the operators in more depth, we utilize a $2+1$-dimensional $\mathbb{Z}_{2}$ gauge theory of the quantum eight-vertex model derived in Appendix [C1]. The degrees of freedom of this gauge theory reside on the links of the square lattice and have a one-to-one correspondence to arrow configurations of the eight-vertex model. The Hamiltonian of the $\mathbb{Z}_{2}$ gauge theory is

$$
H_{\mathrm{q} 8 \mathrm{v}}=H_{\mathrm{V}}+H_{\text {flip }}
$$

where $H_{\text {flip }}$ has the form of a sum over plaquettes of $\mathbb{Z}_{2}$ flip operators ${ }^{8}$ :

$$
H_{\text {flip }}=-\sum_{\vec{x}} \sigma_{1}^{3}(\vec{x}) \sigma_{2}^{3}\left(\vec{x}+\vec{e}_{1}\right) \sigma_{2}^{3}(\vec{x}) \sigma_{1}^{3}\left(\vec{x}+\vec{e}_{2}\right)
$$

The potential energy terms are combinations of operators which project onto the allowed select $a, b$, $c$ and $d$ vertices, and assign the weights $a, b, c$ and $d$ to different contributions to the wave function. The explicit form of the potential energy terms $H_{\mathrm{V}}$ is given in Eq. (C6). As in all $\mathbb{Z}_{2}$ gauge theories [31 57], the physical states of this theory satisfy the constraint of gauge invariance ("Gauss Law")

$$
G(\vec{x})=\sigma_{1}^{1}(\vec{x}) \sigma_{2}^{1}(\vec{x}) \sigma_{1}^{1}\left(\vec{x}-\vec{e}_{1}\right) \sigma_{2}^{1}\left(\vec{x}-\vec{e}_{2}\right)=1 \quad \forall \vec{x}
$$

which in this context simply expresses the restrictions on the configurations allowed in the eight-vertex model. The operator $G(\vec{x})$ is the generator of local time-independent gauge transformations [31, 57].

In Appendix C2 we derive the dual theory of the quantum eight-vertex Hamiltonian of Eq. (4.3). The degrees of freedom of the dual theory are defined on the sites of the dual square lattice. The Hamiltonian is

$$
H_{\mathrm{q} 8 \mathrm{v}, \text { dual }}=H_{\mathrm{V}, \text { dual }}-\sum_{\vec{r}} \tau^{1}(\vec{r})
$$

\footnotetext{
8 Throughout this section and in Appendix C we use the standard notation used in lattice gauge theories [57]. The $\sigma$ 's are Pauli matrices; the superscript is a Pauli matrix label and the subscript indicates the spatial direction, $1=$ horizontal and $2=$ vertical. Notice that the notation used by Kitaev [42] is somewhat different. Please see Appendix C] and figures 8 and 9 for details of the notation that we use here.
} 
where $H_{\mathrm{V} \text {,dual }}$ is given in Eq. (C6) and Eq. (C15). We will use the both the gauge theory and its dual to investigate these phases.

Let us discuss briefly the observables of this model, how they behave in the ordered (confined) and disordered (deconfined) phases and what critical behavior they exhibit at the phase transition lines. As we note in Appendix C2 the quantum eight-vertex model retains the two-sublattice structure of the classical model. The $\mathbb{Z}_{2} \times \mathbb{Z}_{2}$ symmetry of the classical model also survives in the quantum theory.

1. Order and disorder operators: We denote by $\tau(A)$ and $\tau(B)$ the $\tau^{3}$ (order) operator for sublattice $A$ and $B$ respectively. In the gauge theory this operator is a disorder or kink operator [31]; in this 2D gauge theory this is just the flux (monopole) operator of Refs. [57, 97], or the vison operator of Refs. [7, 98]. The order operators are just the order parameters of the sublattice Ising models. As such they acquire an expectation value in the ordered phase (where the staggered polarization has an expectation value as well). In the disordered phase their equal-time correlation functions decay exponentially with distance (as do their connected equal-time correlators in the ordered phase). The precise behavior of this correlation function is only known on the decoupling curves where they reduce to the correlation functions of the 2D classical Ising model, and on the critical lines! The scaling dimension of this spin field (which is known as the "twist" field in conformal field theory [48,96]), is $1 / 8$ not only at the decoupling point (as is well known from the $2 \mathrm{D}$ Ising model) but along the entire phase boundary. We note however, that the spin fields can not be represented directly in terms of the boson, but only in its orbifold [99].

Similarly, we will denote by $\mu(A)$ and $\mu(B)$ the "frustration" (or "fractional domain wall") or disorder operator [32] for sublattices $A$ and $B$ respectively. In the 2D classical theory, this is the dual of the order operators and in conformal field theory it is also represented by a twist field. It has the same scaling dimension as the spin field. On the other hand, in the gauge theory, the disorder operators correspond to a local violation of the Gauss-Law constraint, Eq. (4.13), by imposing that $G(\vec{x})=-1$ just at site $\vec{x}$, i.e. a static source (i.e. an "electrode", or Polyakov loop) at site $\vec{x}$. This operator is analogous to the holon (or "monomer") operator in the Rokhsar-Kivelson quantum dimer model. This expectation value of products of two operators of this type serves as a test for confinement. The disorder operators have a non-zero expectation value in the quantum disordered phase. A simple minded calculation shows that, in in the ordered phase, the ground state with two such defects (or sources) separated at a distance $R$, has a non-zero energy $U(R)$, which grows linearly with $R, U(R) \sim \sigma R$, consistent with the fact that the defects create a fractional domain wall in an ordered state. We expect that the string tension $\sigma$ vanishes as the critical lines are approached, with a behavior dictated by that of the gap scale, i.e., $\xi^{-z}$. Thus, the excitations created by the disordered operator are confined in the ordered phase $^{9}$. In contrast, in the quantum disordered phase, the energy of a pair of defects saturates to a finite value if $R \gg \xi$. Hence, the disordered phase is deconfined.

It is also interesting to ask what is the energy cost of a set of vortex configurations on the quantum critical lines. This can be done in a number of ways. We note here that using pathintegrals the change of the ground state energy can be found by computing the path integral in a background of vortices, normalized by the path-integral without vortices. Consider the simple (an general) case of two vortices of magnetic charges $\pm m$ separated a distance $R$. Let is denote this amplitude by $W(R, T)$, where $T$ is the (infinite) time-span of the system. In the dual gauge theory this is the same as the computation of two static Wilson (or Polyakov) loops, corresponding to two static sources with electric charges $\pm m$ at a distance $R$ from each other.

\footnotetext{
9 The holon operator of the quantum dimer model has the same behavior in both the columnar and staggered phases.
} 
The energy cost of these defects is

$$
U(R)=\lim _{T \rightarrow \infty}\left(-\frac{1}{T} \ln W(R, T)\right)
$$

We can compute $U(R)$ by using the path-integral for the quantum Lifshitz theory of Eq. (2.12) in a background of vortices, which amounts to modifying the action by the minimal coupling shift

$$
\left(\nabla^{2} \phi\right)^{2} \rightarrow(\vec{\nabla} \cdot(\vec{\nabla} \phi-\vec{A}))^{2}
$$

By explicit calculation we find that

$$
U(R)=0
$$

In other terms, the interaction energy of vortices is zero! A simple way to understand this results is to recall that, in imaginary time, the quantum Lifshitz model is a theory of a smectic liquid crystal. The vortex state is just a configuration of screw dislocations running along the $z$ axis (imaginary time). Since the smectic is isotropic and has no resistance to shear in the $x y$ plane, these defects do not cost any energy. From a quantum mechanical point of view this result is consistent with the known fact that at the RK point of the quantum dimer model, the interaction energy of a pair of monomers is zero ${ }^{10}$. In gauge theory language this means that at this critical point vortices are completely free. ${ }^{11}$ In contrast, at quantum critical points of Lorentz invariant gauge theories, which have $z=1$, it is known [100] that at their critical point the effective interaction has the universal law $U(R) \sim 1 / R$, in all dimensions.

2. Polarization: The polarization operator, the local arrow configuration on a link, is the natural order parameter. In terms of the order operators it reads $P=\tau(A) \tau(B)$. As we saw above in the classical "antiferroelectric" ordered phase, $P$ is a natural order parameter, although appropriately-staggered expectation values of $\tau(A)$ and $\tau(B)$ also do not vanish in this ordered phase. The behavior of the polarization operator on the critical lines of the classical model was studied by Kadanoff and Brown [95], who found that its scaling dimension is $\Delta_{P}=1 / 8 \pi \kappa$, with $\kappa$ given by Eq. (4.9). From the results of Appendix $\mathrm{A}$ we see that we can identify the polarization operator with the boson operator $P \sim \cos (\varphi)$ in the quantum Lifshitz theory, which has the same scaling dimension.

3. Mass term: In the classical theory the mass term, or "energy density", is the product of two (dual) spin variables $\tau$ on nearest neighboring sites on the same sublattice. We will denote them by $\mathcal{E}(A)$ and $\mathcal{E}(B)$ respectively. Although in the quantum theory these operators no longer correspond to an energy density, we will define $\mathcal{E}=(\mathcal{E}(A)+\mathcal{E}(B)) / \sqrt{2}$, as usual, and refer to this operator as to the mass term since it drives the theory away from criticality. In Ref. 95] it is found that the scaling dimension of this operator is $\Delta_{\mathcal{E}}=1 / 2 \pi \kappa=4 \Delta_{P}$, which led to the identification $\mathcal{E} \sim \cos (2 \varphi)$. If we regard this operator as acting on the critical line along the $d=0$ axis, i.e. the 6 -vertex model, we see that this is a relevant operator for the quantum Lifshitz model as well. Grinstein's RG arguments [54] show that this perturbation drives the system into a massive phase with a residual unbroken $\mathbb{Z}_{2}$ symmetry. This is just the disordered phase of the quantum eight-vertex model, and this perturbation drives the system towards the Kitaev point. A similar analysis works on the $c^{2}=d^{2}+2$ critical line.

\footnotetext{
${ }^{10}$ We thank Shivaji Sondhi for this remark.

11 This result, Eq. (4.17) holds strictly only at the quantum Lifshitz fixed point (or rather line) fixed point, Eq. (2.12). However, marginally irrelevant operators such as $(\nabla \phi)^{4}$, discussed extensively in Refs. 75 76], give rise to corrections to scaling which change the behavior of $U(R)$ from Eq. (4.17) to a $(\ln R) / R^{2}$ law 75]. We thank T. Senthil for pointing this out to us.
} 
4. Wilson loops: As it is well known gauge theories, the natural test for confinement is the behavior of the Wilson loop operator $W_{\gamma}=\prod_{\ell \in \gamma} \sigma^{3}(\ell)$, where $\{\ell\}$ is a set of links that belong to a closed path $\gamma$ on the (direct) lattice (see Ref. 57] and references therein). Since these operators are off-diagonal in the arrow representation, i.e. in the representation in which $\sigma^{1}$ is diagonal, they do not have an analog in the classical 2D eight-vertex model. A simple argument 31] shows that in the ordered state, which for $c \rightarrow \infty$ is essentially an eigenstate of $\sigma^{1}$, the expectation value of the Wilson loop is zero and that first non-zero contribution comes from acting $N(\gamma)$ times with the flip operator, where $N(\gamma)$ is the number of plaquettes enclosed inside the path $\gamma$, and that the behavior thus found has the form $\exp (-\operatorname{constant} N(\gamma))$, i.e. an area law. Thus the ordered state is a confining phase. Conversely, deep in the disordered phase, the ground state is essentially an eigenstate of the flip operator and we get a perimeter law, i.e. deconfinement.

5. Spinors: The classical 2D eight-vertex model has two Majorana fermion operators $\psi(A)$ and $\psi(B)$, constructed as usual as products of order and disorder operators [32], $\psi \sim \tau \mu$. We can also define an analog of the spinors in the quantum theory (see the discussion in Appendix $\mathrm{A}$ on charge and vortex operators in the $2+1$-dimensional quantum Lifshitz model). The scaling dimension of the spinors is [95] $\Delta_{\psi}=(2 \pi \kappa+1 / 2 \pi \kappa) / 4$, a result familiar from the Luttinger model.

6. Other operators. The classical $2 \mathrm{D}$ eight-vertex model contains a marginal operator $E=$ $\mathcal{E}(A) \mathcal{E}(B)$, with scaling dimension $\Delta_{E}=2$, which is responsible for the lines of fixed points with varying critical exponents. The quantum eight-vertex model discussed here has a similar behavior. In the quantum Lifshitz model the marginal operator is ${ }^{12} E \sim\left(\nabla^{2} \varphi\right)^{2}$. Another operator of interest is the crossover operator $(\mathcal{E}(A)-\mathcal{E}(B)) / \sqrt{2}$ which breaks the sublattice symmetry and has scaling dimension $2 \pi \kappa$, The operator product expansion of two crossover operators generates an operator symmetric under sublattice exchange, and is identified with a charge-two vortex operator. The scaling dimension of this operator is $8 \pi \kappa$ and it is becomes marginal at $\kappa=1 / 4 \pi$, driving the Kosterlitz-Thouless transition along the $d=0$ line. ${ }^{13}$ Other interesting operators are the "mixed" operators $\tilde{P}=\tau(A) \mu(B)$ and $\tilde{P}^{*}=\mu(A) \tau(B)$, both with scaling dimension $\Delta_{\tilde{P}}=\pi \kappa / 2$. Hence $\tilde{P}$ and $\tilde{P}^{*}$ are vortex operators as well; these are non-local with respect to $P$. They have been identified as the holon creation operators in the context of the quantum dimer model [1]].

\section{Another quantum eight-vertex model}

Let us end this section by noting that there is a simpler quantum eight-vertex model that one can write down whose behavior is quite different from the one we have discussed here. Basically, this model will give weights to all the vertices, according to their type. In addition, there will be a flip term. In its dual form the model is

$$
H=\sum_{\vec{r}}\left(\varepsilon_{a} \mathcal{P}_{a}(\vec{r})+\varepsilon_{b} \mathcal{P}_{b}(\vec{r})+\varepsilon_{c} \mathcal{P}_{c}(\vec{r})+\varepsilon_{d} \mathcal{P}_{d}(\vec{r})\right)-\sum_{\vec{r}} \tau^{1}(\vec{r})
$$

where the projection operators are defined in Eq. (C15). The conventional Baxter weights of the classical model (i.e. when the flip term is absent) are $a=e^{-\frac{\varepsilon_{a}}{k_{B} T}}$, etc.

\footnotetext{
12 As we noted above, on symmetry grounds a lattice model may generate additional operators which break rotational invariance, absent at this RK point, which may drive the transitions to be first order, see 7576 .

13 Notice that the charge-one vortex operator is not allowed in this case, which is the operator driving the KT transition in the 2D classical XY model 101102 103 104].
} 
We first note that this model is not of the Rokhsar-Kivelson type (except when $\varepsilon_{a}=\varepsilon_{b}=\varepsilon_{c}=\varepsilon_{d}$, which is a dual version of the Kitaev model). The ground state wave function does not look like the statistical weights of the classical two-dimensional model, i.e. it is not a linear superposition of states weighted according to the vertices present. Thus, strictly speaking we can not use the machinery of this paper to study the ground state of this model. We do expect, however, that this model will have the same ordered phases, just like the ferro-electric and anti-ferro-electric phases of the eight-vertex model, as well as the same confinement-deconfinement properties discussed above. In addition, we expect that these ordered phases have the same spectrum and general properties as the ones we found in the Rokhsar-Kivelson type model we studied above. Similarly, in the regime where the flip term dominates, the ground state of this model is a uniform quantum disordered state, with the same properties as those of the topological phase of the model studied here.

However, the quantum critical properties of Eq. (4.18) are completely different from the quantum eight-vertex model discussed in the rest of this section. They instead have a number of features in common with well-known three-dimensional classical models. By setting $\varepsilon_{a}+\varepsilon_{b}=\varepsilon_{c}+\varepsilon_{d}$, this model reduces to two interpenetrating two-dimensional Ising models in a transverse field, as the four-body interactions cancel. Thus along these curves this model has second-order phase transitions in the universality class of the classical three-dimensional Ising model, as opposed to the quantum eightvertex model discussed above. The latter has $z=2$, while the model of Eq. (4.18) therefore has dynamical exponent $z=1$ and is Lorentz invariant in the critical regime. It is easy to see that this behavior extends beyond the decoupling point. Indeed, the universality class of the 3D classical Ising model is controlled by the Wilson-Fisher fixed point and is accessible by the $4-\epsilon$ expansion. Away from the decoupling point we have then two 3D Ising models which are coupled through their energy density. This theory is in the universality class of a two-component real field $\left(\varphi_{1}, \varphi_{2}\right)$, whose $U(1)$ symmetry is explicitly broken down to a discrete $\mathbb{Z}_{2} \times \mathbb{Z}_{2}$ symmetry by quartic operators of the form $\varphi_{1}^{2} \varphi_{2}^{2}$. This is a cubic symmetry-breaking perturbation and it is well known to be perturbatively relevant at the Wilson-Fisher fixed point of the two decoupled Ising models, but irrelevant at the Wilson-Fisher fixed point for the classical $X Y$ model $^{14}$. It is well known from perturbative $4-\epsilon \mathrm{RG}$ studies that the transition near the decoupling point that the transition may also become a fluctuation-induced first order transition depending on the sign of the effective coupling (see for instance Refs. [3] 105]).

\section{NON-ABELIAN TOPOLOGICAL STATES}

In this section we broaden our scope and discuss field theories with continuous non-abelian symmetries. One can of course obtain a quantum critical point with non-abelian symmetries by taking copies of the quantum Lifshitz Hamiltonian. By choosing the charge lattice of the vertex operators carefully one can describe a theory with a non-abelian symmetry, say $S U(N)$. However the equivalent conformal field theories thus obtained always have integer central charge and are at level 1. Although critical, such theories only support excitations with abelian statistics. One may try to attempt to generalize this result to models by writing down a theory with more structure, such as one related to a Wess-Zumino-Witten model. However, we will find that, contrary to naive expectation, such theories will turn out not to be critical but, instead, in a phase with a finite gap, a topological phase, just like the fractional quantum Hall effect. We will see that it is not at all clear how to find a conformal quantum critical point with a non-trivial non-abelian symmetry.

Nevertheless it is possible to use the approach of the previous sections to discuss topological nonabelian phases, even if any associated non-trivial quantum critical points are not yet known or do not exist. By analogy with the theory of fractional quantum Hall states, as well as from general results

14 This is a classic example of a dangerously-irrelevant operator, since it removes the would-be Goldstone boson from the broken-symmetry phase. 
in Chern-Simons gauge theory, one expects that a time-reversal-breaking topological phase in $2+1$ dimensions should have an effective field theory description in terms of a Chern-Simons gauge theory at some level $k$. On closed manifolds this is a topological field theory in the sense that the partition function is independent of the metric of 2+1-dimensional space-time, and that the expectation values of its gauge-invariant observables, the Wilson loops, depend only on the topology of the loops such as the knot invariants [26].

By analogy with the approach pursued in the previous sections, we will discuss the properties of the wave functionals of this theory. This will lead us to consider first the strong-coupling limit of Yang-Mills theory with a Chern-Simons term, and later the topological sector of this theory. We will find the ground-state wave functional of this theory, and show how it is related to a two-dimensional Wess-Zumino-Witten (WZW) model for a specific choice of polarization (i.e., the choice of canonically conjugate variables) which is natural in the strong coupling limit of the Yang-Mills Chern-Simons theory. Even though the WZW model is a conformal field theory with algebraically-decaying correlators, the gauge-invariant ground-state correlators in the two-dimensional quantum theory are exponentially decaying. Topological invariance will only be attained by correlators of gauge-invariant operators, Wilson and Polyakov loops, and only at long distances. The excitations obey non-abelian statistics: when bringing particles around one another, not only does one pick up phases, but the order in which they are exchanged affects the final state.

We will also study a doubled Chern-Simons theory which, contrary to the fractional quantum Hall effect, is time-reversal invariant. Using by now standard methods, we will construct the ground-state wave functionals of this doubled theory for a gauge group $G$ at level $k$, and use its connection with a gauged WZW model to understand its properties. We note that the doubled theory is often used to circumvent technical problems involved in the use of the formally anomalous wave functional of the undoubled theory: see the discussion in the subsections $\mathrm{VB}$ and $\mathrm{VC}$. The topological properties of the doubled theory have been recently discussed in depth by Freedman et al. [50].

\section{A. Field theories with continuous non-abelian symmetries}

Let us attempt first to construct a non-abelian version of the quantum Lifshitz theory, which one might hope would still be a quantum critical theory. We will follow the same procedure as in the abelian case. Since there are many two-dimensional Euclidean critical points with non-abelian symmetry, the first thing to try is the above procedure for making a two-dimensional quantum theory from a twodimensional classical theory. This procedure is indeed fairly general. One can easily construct a set of a appropriate projectors $Q_{i}$ in a lattice model without local constraints. However, the caveat "without constraints" is quite important: many interesting classical lattice models have local constraints like in the dimer model and in the six- and eight-vertex models. This means that one must find a flip operator which respects the constraints. There is no guarantee that there is a set of flips which are both local and ergodic. This is particularly apparent in models where the two-dimensional degrees of freedom are closed loops. At a critical point, arbitrarily-long loops are an important part of the configuration space, so one must construct some sort of flip which still acts non-trivially but locally on these loops. In the continuum limit, it is easy to imagine such flips, but it is not always obvious how to make them act consistently on the lattice. Nevertheless, the Rokhsar-Kivelson Hamiltonian and the quantum eight-vertex model provide examples of models where one can solve this problem.

For field theories, the issue is similar. If a theory has an action $S_{2 d}$ and one ignores constraints such as gauge invariance, one can easily find projection operators $Q(x)$ which annihilate the state weighted by $e^{-S_{2 d}}$. For a single field $\varphi$, we have

$$
Q=\frac{\delta}{\delta \varphi}+\frac{\delta S_{2 d}}{\delta \varphi}
$$

and the Hamiltonian is defined via Eq. (2.9). This $Q$ is hardly unique: one can multiply it on the left 
by any operator without changing the ground state. It is important to note that this construction is not always easy to implement. Many interesting two-dimensional field theories do not have a simple-todescribe action: they are usually defined instead in terms of representations of an infinite-dimensional symmetry algebra such as the Virasoro algebra, arising from conformal invariance [48]. For example, the action of a $G / H$ coset model (e.g. a conformal minimal model) is written as a gauged WZW model such as we will discuss below; one must then worry about the constraints of gauge invariance.

A point we wish to emphasize, however, is that even if one succeeds in constructing such a Hamiltonian, the 2+1-dimensional theory may be qualitatively very different from the classical two-dimensional theory. For example, the classical theory may be critical and have algebraically-decaying correlators, but the quantum theory may have exponentially-decaying correlators in the ground state. The reason is that quantum-ground-state correlators are weighted by $\left|\Psi_{0}\right|^{2}$, not $\Psi_{0}$. For the models discussed above, this has effects which are easy to take account of: it is the reason that the axes in figure 5 are labeled by $c^{2}$ and $d^{2}$ instead of $c$ and $d$. If however $\Psi_{0}$ has a complex part, the effects can be much more dramatic.

Let us first discuss the simplest two-dimensional classical field theories with a non-trivial nonabelian symmetry. The $G$ principal chiral model is written in terms of a unitary matrix field $g$ taking values in some simple Lie group $G$, with action

$$
S_{P C M}=-\frac{1}{8 \pi u} \int d^{2} x \operatorname{Tr}\left[g^{-1} \partial^{a} g g^{-1} \partial_{a} g\right] .
$$

This action has a global $G_{L} \times G_{R}$ symmetry under $g \rightarrow L g R^{\dagger}$, where $L$ and $R$ are elements of separate groups dubbed $G_{L}$ and $G_{R}$. This model looks critical: the coupling constant $u$ is naively dimensionless. However, in two dimensions, the one-loop beta function is proportional to the curvature of the targetspace manifold $G$. Simple Lie groups $G$ are curved: for example, $S U(2)$ as a manifold is isomorphic to a three-sphere. Moreover, one finds that the trivial fixed point at $u=0$ is unstable, and the model has a finite correlation length proportional to an exponential in $1 / u$. Correlators decay exponentially, not algebraically. The phenomenon where a naively dimensionless coupling becomes dimensionful due to loop corrections is known in the particle physics literature as dimensional transmutation; it is familiar in condensed-matter physics in the Kondo problem and the Hubbard model at half-filling. Another reason a gap should appear is that the Mermin-Wagner-Coleman theorem does not allow Goldstone bosons in two Euclidean dimensions. One would obtain $S_{P C M}$ in a theory as the low-energy limit of a fermionic theory where one attempts to spontaneously break the chiral symmetry $G_{L} \times G_{R}$ to its diagonal $G_{D}$ subgroup. The would-be Goldstone bosons would take values on the manifold $G_{L} \times G_{R} / G_{D} \cong G$. This satisfies the theorem by having the low-energy excitations get a mass and restoring the symmetry to the full $G_{L} \times G_{R}$.

A critical theory with non-abelian symmetry $G$ does occur when an extra term, the Wess-Zumino term, is added to $S_{P C M}$. This term is easiest to write in a three-dimensional space $M$ which has the two-dimensional space of interest as a boundary; we assume the two-dimensional space has no boundary. It is

$$
\Gamma(g)=\frac{1}{12 \pi} \int_{M} d^{3} x \epsilon^{\alpha \mu \nu} \operatorname{Tr}\left[g^{-1} \partial_{\alpha} g g^{-1} \partial_{\mu} g g^{-1} \partial_{\nu} g\right]
$$

One finds that different ways of extending space to three dimensions result only in changing $\Gamma(g)$ by $2 \pi$ times an integer. Thus we can add $i k \Gamma(g)$ to $S_{P C M}$ for any integer $k$. This model has a critical point at $u=1 /|k|[106]$. This theory, with action

$$
I(g)=S_{P C M}(g, u=1 /|k|)-i k \Gamma(g),
$$

has conformal invariance and is known as the $G_{k}$ Wess-Zumino-Witten (WZW) model. A great deal of information is known about the WZW model; the most important for our current purposes is that the correlators decay algebraically, as they must at a critical point. 
Since the WZW model has an explicit action, it is easy to use the trick in Eq. (5.1) to find a two-dimensional Hamiltonian which has a ground-state wave functional with amplitudes given by the WZW action, i.e.,

$$
\Psi_{0}[g]=e^{-I(g)} .
$$

The WZW model is critical, so one might guess that the quantum version with this wave function would also be critical, in the fashion of the theories in the previous sections. However, it is not. The reason is that the Wess-Zumino term has an $i$ in front of it, so

$$
\left|\Psi_{0}[g]\right|^{2}=e^{-2 S_{P C M}(g, u=1 /|k|)}
$$

Thus equal-time correlators in the ground state are weighted with the action of a theory with a finite correlation length, the principal chiral model. These correlators decay exponentially.

We do note that when the level $k=1$, one can use an alternate method to find the $2 \mathrm{~d}$ WZW correlators in the ground-state of a quantum theory. All the fields of $S U(N)_{1}$ can be written in terms of $N-1$ free bosons [48]. The combination of the corresponding quantum Lifshitz theories will then have the $S U(N)_{1}$ correlators in its ground state. Likewise, $O(N)_{1}$ and $S U(2)_{2}$ can be written in terms of free Majorana fermions. Since the different bosons or fermions here do not interact, the physics is the same as that discussed earlier; in particular the model is essentially free, and the excitations are abelian.

\section{B. Yang-Mills, Chern-Simons, and Wess-Zumino-Witten}

Since it is not possible to have a wave functional with $|\Psi[g]|^{2}=e^{-I[g]}$, we will need to work harder to find how the physics of the WZW model can arise in $2+1$ dimensions. A connection between the WZW model and the wave functions of $2+1$-dimensional Chern-Simons gauge theory has long been known [26,107]. Chern-Simons theory without any matter fields is a topological field theory: the physical states are Wilson and Polyakov loops, and their correlation functions do not depend on distance but only on topological properties of the loops. These correlators can be expressed in terms of the Verlinde numbers, which also describe the (chiral) fusion rules of the WZW model [108]. These topological field theories have been discussed in detail in many places. One discussion of issues closely related to those of interest here is the recent work of Freedman et al. [50]; we will discuss the relation of our results to this paper in the next subsection.

In this subsection we will describe a wave functional which after squaring and averaging over fields indeed yields the WZW partition function. We will show that this wave functional is the ground state of a 2+1-dimensional gauge theory. This all sounds like exactly what we want for a quantum critical point, but in a gauge theory, physical observables must be gauge invariant. Gauge-invariant states will turn out to have a gap, and gauge-invariant correlators in the ground state will be exponentially decaying. Introducing a gauge field therefore will not yield a quantum critical point with non-abelian symmetry. It will yield, however, something just as interesting: an explicit Hamiltonian for a theory in a topological phase.

Before discussing construction of these wave functionals, which was done in great detail by Witten 52] and whose construction we will follow here, it is worthwhile to discuss first its physical meaning

and to emphasize some well-known facts of Chern-Simons gauge theories [26]. The ground state wave function of any field theory can be viewed as the quantum mechanical amplitude of some arbitrary state into the vacuum (or ground state), i.e. what we normally call the vacuum state in a given representation. As such this amplitude is the functional integral of the quantum field theory on an open manifold bounded by the initial time surface. However, the partition function of a topological gauge theory such as Chern-Simons is only gauge invariant on a manifold without boundary. Thus, the ground state wave function has a gauge anomaly. Nevertheless, if the wave function is used to 
compute expectation values of gauge-invariant observables, the result is gauge invariant. The reason is that the computation of expectation values (as well as all inner products) involves the conjugate wave function, i.e. the amplitude to evolve from the vacuum state in the remote past into the chosen state at the fixed time surface. Consequently, the gauge anomaly cancels in the computation of the expectation values of gauge-invariant operators. In this way, at a formal level, the computation of expectation values leads to a formally "doubled" theory even though the number of degrees of freedom has not changed. In subsection $\nabla \mathrm{V}$ we will discuss a physically doubled theory which has a formal relation with what we do in this Subsection. There is an extensive literature on the technical issues involved in this problem: the relevant discussion for the analysis done in this paper can be found in Refs. 52 109, 110.111.

We should note here that the gauge anomaly of the wave function is formally (mathematically) analogous to the gauge anomaly of Chern-Simons theory on manifold with a spatial edge. In the latter, the anomaly is physical: to cancel the anomaly the gauge-invariant theory must include physical degrees of freedom residing at this $1+1$-dimensional boundary. In the incompressible fractional quantum Hall fluid, this results in physical edge states [112.113.

Another important fact is that, since Chern-Simons gauge theory is a topological field theory, the partition function on a closed space-time manifold is independent of the metric. However, the boundary of the manifold and the choice of polarization break the general coordinate invariance of Chern-Simons theory. In particular, the choice of holomorphic polarization induces a conformal structure in the wave functional [26, 107] which is absent in other polarizations 114]. We will see that the conformal wave function arises naturally in a specific theory, the strong-coupling limit of the Yang-Mills Chern-Simons theory.

The wave functional of interest was discussed in depth by Witten [52]. It involves the WZW field $g$ coupled to a gauge field $A_{i}$ taking values in the Lie algebra of $G$. It is most convenient to write the gauge field in complex coordinates $A_{z} \equiv\left(A_{1}-i A_{2}\right) / 2$ and $A_{\bar{z}} \equiv\left(A_{1}+i A_{2}\right) / 2$. When the symmetry $G_{R}$ of the WZW model is gauged, the closest thing to a gauge-invariant action is

$$
I(g, A)=I(g)+\frac{k}{4 \pi} \int d^{2} z \operatorname{Tr}\left[2 A_{\bar{z}} g^{-1} \partial_{z} g-A_{\bar{z}} A_{z}\right]
$$

This action is not gauge invariant; the subgroup $G_{R}$ of $G_{L} \times G_{R}$ is anomalous [52]. Indeed, the fields under gauge transformations $U(z, \bar{z})$ in $G_{R}$ as

$$
g \rightarrow g U, \quad A_{i} \rightarrow U^{-1} A_{i} U+U^{-1} \partial_{i} U
$$

so that the action $I(g, A)$ transforms as

$$
I(g, A) \rightarrow I(g, A)+\frac{k}{4 \pi} \int d^{2} z \operatorname{Tr}\left[A_{z} U \partial_{\bar{z}} U^{-1}-A_{\bar{z}} U \partial_{z} U^{-1}\right]-i k \Gamma(U) .
$$

Note, however, that the variation of $I(g, A)$ under gauge transformations is independent of $g$. If we were to simply square a wave functional proportional to $e^{-I(g, A)}$, the WZW term in the action would cancel and we would be back a gauged principal chiral model. Instead, we define a wave functional depending only on $A$ by doing the path integral over $g$ :

$$
\Psi[A] \equiv \int[D g] e^{-I(g, A)}
$$

This wave functional does yield the full WZW partition function after squaring and integrating over $A[52]$. We define this integrated $|\Psi|^{2}$ as

$$
|\Psi|^{2} \equiv \frac{1}{\operatorname{vol} \hat{G}} \int[D A]|\Psi[A]|^{2},
$$


where we have divided the measure by the volume of the gauge group $\hat{G}$ because it follows from Eq. (5.9) that even though $\Psi[A]$ is not gauge-invariant, $\overline{\Psi[A]} \Psi[A]$ is. Substituting the definition Eq. (5.10) gives

$$
\begin{aligned}
|\Psi|^{2} & =\frac{1}{\operatorname{vol} \hat{G}} \int[D A][D g]\left[D h^{-1}\right] e^{-I(g, A)-\left(I\left(h^{-1}, A\right)\right)^{*}}, \\
I(g, A)+\left(I\left(h^{-1}, A\right)\right)^{*} & =I(g)+I(h)+\frac{k}{2 \pi} \int d^{2} z \operatorname{Tr}\left[A_{\bar{z}} g^{-1} \partial_{z} g-A_{z} \partial_{\bar{z}} h \cdot h^{-1}-A_{\bar{z}} A_{z}\right]
\end{aligned}
$$

Note that we have defined $A$ so that $\left(A_{z}\right)^{\dagger}=-A_{\bar{z}}$, i.e. covariant derivatives have no $i$ in them. We have also used the easily-proven fact that $I(h)=I^{*}\left(h^{-1}\right)$. Since the integral over $A$ is Gaussian, it can easily be done, giving

$$
|\Psi|^{2}=\frac{1}{\operatorname{vol} \hat{G}} \int[D g]\left[D h^{-1}\right] \exp \left(-I(g)-I(h)+\frac{k}{2 \pi} \int d^{2} z \operatorname{Tr}\left[g^{-1} \partial_{z} g \partial_{\bar{z}} h \cdot h^{-1}\right]\right) .
$$

This can be simplified by using the Polyakov-Wiegmann formula 115]

$$
I(g h)=I(g)+I(h)-\frac{k}{2 \pi} \int d^{2} z \operatorname{Tr}\left[g^{-1} \partial_{z} g \partial_{\bar{z}} h \cdot h^{-1}\right] .
$$

Thus the integrand depends only on the product $f \equiv g h$, so we can change variables to $f$, which cancels the volume of the gauge group. This yields the final expression for the normalization 52]

$$
|\Psi|^{2}=\int[D f] e^{-I(f)}
$$

Thus, remarkably, the integrated square of the wave functional $\Psi[A]$ ends up giving the full WZW path integral, including the imaginary piece: because of the path integral, the right-hand-side of Eq. (5.13) is positive and real as it must be.

To reemphasize a point made earlier, (5.13) does not mean we have now found a $2+1$-dimensional quantum critical point whose equal-time correlators are those of the WZW model. We must first find a Hamiltonian which has $\Psi[A]$ in Eq. (5.10) as its ground state. Once having found that, the physicallyrelevant operators are only those satisfying the proper gauge-invariance properties and regularization. After having done so, we will end up seeing that despite sharing some key properties with the WZW model, the $2+1$-dimensional model is gapped and has exponentially-decaying correlators.

To find such a Hamiltonian, let us go back to the definitions Eq. (5.7) and Eq. (5.10) of the wave functional $\Psi[A]$. Because the only dependence on $A_{z}$ is through the quadratic term, we have

$$
\left(\frac{\delta}{\delta A_{z}}-\frac{k}{4 \pi} A_{\bar{z}}\right) \Psi[A]=0 .
$$

With a little more work [52], one also can show that

$$
\left(D_{\bar{z}} \frac{\delta}{\delta A_{\bar{z}}}+\frac{k}{4 \pi} D_{\bar{z}} A_{z}-\frac{k}{2 \pi} F_{\bar{z} z}\right) \Psi[A]=0
$$

where we have define the covariant derivatives via $D_{i} g=\partial_{i} g-g A_{i}$ and $D_{i} A_{j}=\partial_{i} A_{j}+\left[A_{i}, A_{j}\right]$. The field strength is defined as $F_{\bar{z} z}=\partial_{\bar{z}} A_{z}-\partial_{z} A_{\bar{z}}+\left[A_{\bar{z}}, A_{z}\right]$.

We would therefore like to find a Hamiltonian with a ground-state wave function satisfying Eq. (5.14) and Eq. (5.15). As noted in Ref. [52], these equations arise in the canonical quantization of Chern-Simons theory [107], so this suggests we look there. The precise Hamiltonian turns out to be given by the strong-coupling limit of Yang-Mills theory with a Chern-Simons term [110]. In the action, 
we have a gauge field $A_{\mu}$ taking values in the Lie algebra of $G$; here $\mu=0,1,2$ and $A_{\mu}$ depends on space and time. The action for the strong-coupling limit of Yang-Mills theory on a three-manifold $M$ includes only the electric-field term, namely

$$
S_{S C}=\frac{1}{2 e^{2}} \int_{M} \operatorname{Tr}\left[F_{0 i} F^{0 i}\right] .
$$

This term is not Lorentz-invariant, but does preserve two-dimensional rotational symmetry. The Chern-Simons term is

$$
S_{C S}=\frac{k}{4 \pi} \int_{M} \epsilon^{\mu \nu \alpha} \operatorname{Tr}\left[A_{\mu} \partial_{\nu} A_{\alpha}+\frac{2}{3} A_{\mu} A_{\nu} A_{\alpha}\right]
$$

Under gauge transformations $U(x)$ belonging to $G$, the integrand in $S_{S C}$ is invariant, but the integrand in $S_{C S}$ is not:

$$
S_{C S} \rightarrow S_{C S}+\frac{k}{4 \pi} \int_{M} \epsilon^{\mu \nu \alpha} \operatorname{Tr}\left[\partial_{\mu}\left(A_{\alpha}+U \partial_{\nu} U^{-1}\right)\right]+k \Gamma(U) .
$$

For the manifold $M$ a three-sphere, the latter term turns out to be the winding number of the gauge transformation $U(x)$, and is an integer times $2 \pi k$ [116]. The Chern-Simons term is gauge-invariant if $M$ has no boundary, and $k$ is an integer. If $M$ has a spatial boundary, one must include massless chiral fermions on the edge to restore gauge invariance, giving for example the famous edge modes in the fractional quantum Hall effect [5].

The Hamiltonian with (5.10) as a ground-state wave functional comes from canonically quantizing the theory with action

$$
S=S_{C S}+S_{S C}
$$

following, for instance, Refs. 107, 110,116. Since this is a fairly standard computation, we will be brief here. The gauge invariance allows us to fix temporal gauge $A_{0}=0$, so that the degrees of freedom are the gauge fields $A_{1}$ and $A_{2}$. Their canonical momenta are

$$
\Pi_{i}=\frac{1}{e^{2}} F_{0 i}+\frac{k}{8 \pi} \epsilon_{i j} A_{j} .
$$

The Chern-Simons term contributes nothing to the Hamiltonian, because all the terms are first-order in time derivatives. The classical Hamiltonian in the strongly-coupled limit is therefore

$$
H=\frac{1}{e^{2}} \int d^{2} x \operatorname{Tr}\left[\left(F_{0 i}\right)^{2}\right]=e^{2} \int d^{2} x \operatorname{Tr}\left[\left(\Pi_{i}-\frac{k}{8 \pi} \epsilon_{i j} A_{j}\right)^{2}\right] .
$$

Expanding $A$ and $F$ in terms of generators $T^{a}$ of the Lie algebra of $G$, we impose the canonical commutation relations

$$
\left[A_{i}^{a}(\vec{x}), \Pi_{j}^{b}(\vec{y})\right]=i \delta_{i j} \delta^{a b} \delta^{(2)}(\vec{x}-\vec{y}) .
$$

One of the effects of the Chern-Simons term is that $A_{1}$ and $A_{2}$ do not commute. In the Schrödinger picture, $\Pi_{j}$ is given by

$$
\Pi_{j}=-i \frac{\delta}{\delta A_{j}}
$$

operating on the wave functionals. 
The Hamiltonian of this theory in the Schrödinger picture has a very simple form. Because $F_{0 i}$ includes $\Pi_{i}$, one has the usual ordering ambiguity in the quantum Hamiltonian. We define

$$
E=\frac{i}{e^{2}}\left(F_{01}+i F_{02}\right)=\frac{\delta}{\delta A_{z}}-\frac{k}{4 \pi} A_{\bar{z}}
$$

so that

$$
\left[E^{a}(\vec{x}),\left(E^{b}(\vec{y})\right)^{\dagger}\right]=\frac{k}{2 \pi} \delta^{a b} \delta^{(2)}(\vec{x}-\vec{y}) .
$$

The operators $E$ and $E^{\dagger}$ are like annihilation and creation operators. We then normal-order the Hamiltonian as in section 1 subtracting the vacuum energy to give

$$
H=e^{2} \int d^{2} x \operatorname{Tr}\left[E^{\dagger}(\vec{x}) E(\vec{x})\right] .
$$

Thus the Hamiltonian of strongly-coupled Yang-Mills theory with a Chern-Simons term is precisely of the form Eq. (2.9), like all the others discussed in this paper. Moreover, the annihilation relation $E \Psi[A]=0$ here is identical to the relation Eq. (5.14). We thus have found an explicit Hamiltonian whose ground state obeys the first of the two relations satisfied by $\Psi[A]$ above.

Finding the Hamiltonian alone does not complete the canonical quantization of gauge theories with a Chern-Simons term. Fixing the gauge $A_{0}=0$ still allows time-independent gauge transformations. Moreover, there is no time derivative $\dot{A}_{0}$ in Eq. (5.19), so $A_{0}$ should be viewed as a Lagrange multiplier which results in a constraint. When we are canonically quantizing the theory in $A_{0}=0$ gauge, this constraint is implemented on the wave functions. Specifically, one has

$$
\left(D_{\bar{z}} \frac{\delta}{\delta A_{\bar{z}}}+D_{z} \frac{\delta}{\delta A_{z}}+\frac{k}{4 \pi} \partial_{z} A_{\bar{z}}-\frac{k}{4 \pi} \partial_{\bar{z}} A_{z}\right) \Psi[A]=0 .
$$

This operator is the generator of time-independent gauge transformations on $A_{z}$ and $A_{\bar{z}}$, so this condition amounts to requiring that the wave function be invariant under such transformations. Another way of viewing this constraint is as requiring that the wave functionals obey the non-abelian version of Gauss' Law. Requiring Gauss' Law along with Eq. (5.14) yields Eq. (5.15), the other desired relation: adding Eq. (5.14) and Eq. (5.15) together yields Eq. (5.27). Moreover, the fact (5.9) that $\Psi[A]$ is not gauge invariant is precisely the effect of the fact (5.18) that the Chern-Simons action is not gauge invariant when there are boundaries [107]; one can think of the constant-time slice required to define a wave functional as a boundary in space time.

We have seen that the wave functional Eq. (5.10) indeed describes a zero-energy ground state of the $2+1$-dimensional theory with action Eq. (5.19). We now need to understand the correlators in the ground state and the excited states. Luckily, the former issue has been studied in Refs. 109 117, 118 119], and the latter in Refs. [110 111]. We will show in the next subsection that the ground-state are those of a topological field theory, and that one can prove there is a gap in the spectrum.

\section{The doubled theory}

It is both convenient and physically important to study the wave function of the "doubled" theory, where we have two gauge fields $A$ and $B$. The three-dimensional action is the sum of an action of the form of Eq. (5.19) for both fields, where the Chern-Simons term for the field $A$ has coefficient $k$, while that for $B$ has coefficient $-k$. As opposed to Chern-Simons theory with a single field, the doubled theory is also invariant under time reversal and parity, if the fields $A$ and $B$ are exchanged under these 
transformations ${ }^{15}$. The two fields are not coupled in the action, so formally the wave functional of the doubled theory factorizes:

$$
\chi[A, B] \propto \Psi[A] \overline{\Psi[B]}
$$

There is also a compelling technical reason to study the doubled theory. We will show that the doubled theory amounts to gauging a non-anomalous symmetry. In particular, after integrating out one of the gauge fields (a simple Gaussian integration), the wave functional is gauge invariant in the remaining field. The theory can be quantized consistently; effectively the regularization (the measure of the path integral) couples the two copies. Thus in spite of this factorization of the wave function, the operators that create the physical states are made of operators acting on each sector, carefully glued together to satisfy the requirements of gauge invariance.

Canonically quantizing the doubled theory is straightforward, since the action splits into decoupled pieces. The ground-state wave functional of the doubled theory can be written as [52]

$$
\chi[A, B] \equiv \int[D g] e^{-I(g, A, B)}
$$

where

$$
I(g, A, B) \equiv I(g)+\frac{k}{4 \pi} \int d^{2} z \operatorname{Tr}\left[2 A_{\bar{z}} g^{-1} \partial_{z} g+2 B_{z} g \partial_{\bar{z}} g^{-1}-A_{\bar{z}} A_{z}-B_{\bar{z}} B_{z}+2 B_{z} g A_{\bar{z}} g^{-1}\right]
$$

One can prove that $\chi[A, B] \propto \Psi[A] \overline{\Psi[B]}$ indirectly by showing that $\chi[A, B]$ satisfies both (5.14) and (5.15) for $A$, and the conjugate equations for $B$. Directly, we prove this by first noting that the Polyakov-Wiegmann identity (5.12) yields

$$
I(g, A)+(I(h, B))^{*}=I\left(h^{-1} g, A, B\right)-\frac{k}{2 \pi} \int d^{2} z \operatorname{Tr}\left[\left(g D_{\bar{z}} g^{-1}\right)\left(h D_{z} h^{-1}\right)\right] .
$$

where the covariant derivatives are $D_{\bar{z}} g^{-1}=\partial_{\bar{z}} g^{-1}+A_{\bar{z}} g^{-1}$ and $D_{z} h^{-1}=\partial_{z} h^{-1}+B_{z} h^{-1}$. In the path integral we can split apart the last term by introducing an auxiliary gauge field $C$ :

$$
\begin{aligned}
\Psi[A] \overline{\Psi[B]}= & \int[D g]\left[D h^{-1}\right] e^{-I(g, A)-(I(h, B))^{*}} \\
= & \int[D g]\left[D h^{-1}\right][D C] \exp \left(-I\left(h^{-1} g, A, B\right)\right. \\
& \left.\quad-\frac{k}{2 \pi} \int d^{2} z \operatorname{Tr}\left[\alpha C_{z}\left(g D_{\bar{z}} g^{-1}\right)+\alpha C_{\bar{z}}\left(h D_{z} h^{-1}\right)+\alpha^{2} C_{z} C_{\bar{z}}\right]\right),
\end{aligned}
$$

where $\alpha$ is a (small) coupling constant. The terms linear in $C$ can be removed by redefining $g$ and $h$. Namely, under $g \rightarrow g+\delta g$ for small $\delta g$, the action

$$
I(g, A) \rightarrow I(g, A)+\frac{k}{2 \pi} \int d^{2} z \operatorname{Tr}\left[g D_{\bar{z}} g^{-1} \partial_{z}\left(\delta g g^{-1}\right)\right] .
$$

Thus if we redefine the fields $g$ and $h$ so that $\delta g$ and $\delta h^{-1}$ obey $\alpha C_{z}=\partial_{z}\left(\delta g g^{-1}\right)$ and $\alpha C_{\bar{z}}=$ $-\partial_{\bar{z}}\left(h \delta h^{-1}\right)$, the mixed terms cancel and the field $C$ decouples. The integrand then only depends on the combination $h^{-1} g$, so changing variables to $f=h^{-1} g$ yields $\chi[A, B]$, up to a factor of the volume of the gauge group:

$$
\Psi[A] \overline{\Psi[B]}=\int[D g]\left[D h^{-1}\right] e^{-I\left(h^{-1} g, A, B\right)}=\operatorname{vol} \hat{G} \chi[A, B]
$$

${ }^{15}$ For this reason, this theory has arisen for example in theories of quantum computation and in superconductivity [50 51], and in effective "coset" field theories of the non-abelian fractional quantum Hall states [120 121 122]. 
To understand this wave functional $\chi[A, B]$, we compute its integrated norm. One can easily do one of the two functional integrals over the gauge fields in the same manner as before, yielding 52 ]

$$
\int[D B]|\chi[A, B]|^{2}=\int[D g] e^{-I_{G / G}(g, A)}
$$

where $I_{G / G}$ is the action of the $G / G$ gauged WZW model, namely

$$
I_{G / G}(g, A)=I(g)+\frac{k}{2 \pi} \int d^{2} z \operatorname{Tr}\left[A_{\bar{z}} g^{-1} \partial_{z} g+A_{z} g \partial_{\bar{z}} g^{-1}-A_{\bar{z}} A_{z}+A_{z} g A_{\bar{z}} g^{-1}\right] .
$$

As opposed to $I(g, A)$ in Eq. (5.7), this action is gauge invariant, because one is gauging the full $G_{L} \times G_{R}$ symmetry of the WZW model. This means the path integral of the doubled theory is well defined and free of anomalies, as well as parity-invariant.

Let us compare the doubled theory to the undoubled one. For the undoubled theory, we found that the effective partition function $|\Psi|^{2}$, the ground-state wave functional squared and integrated over the (one) gauge field, was that of the WZW model. However, constructing excited states and operators directly in the undoubled theory appears to be problematic due to the gauge anomaly discussed above. Although it would seem that a priori one could not require that operators be gauge invariant if the ground-state wave functional itself is not, as we emphasized in Subsection VB from general considerations of Chern-Simons theory we know that the physical observables are gauge invariant, that only gauge invariant observables must be considered, and that their expectation values are free of any anomalies. However, while this is apparent in the path-integral construction of the quantum theory, it is not so apparent if one is to use the wave function, i.e. in terms of a chiral Euclidean WZW model. As we discussed above, at this level one is led to introduce a formal "doubled" theory even for the undoubled theory. Thus, although the wave function itself factorizes the physical states cannot be constructed in terms of arbitrary factors from each sector of the doubled theory. Hence, the requirement of gauge invariance can spoil the apparent factorization suggested by the wave function $\chi[A, B]$ of Eq. (5.29).

The formally correct way to define the correlators in the undoubled theory is in the doubled theory: one can always introduce another field $B$ and then integrate it out. The reason for doing this is that the doubled theory can be properly regulated, because (5.33) is gauge invariant. All the formal manipulations done above are well founded, because the path integral can be defined properly. In contrast in a truly doubled theory, the gauge-invariant physical observables couple to both fields which must then be regarded as genuine degrees of freedom. In contrast, in the undoubled theory, the additional degrees of freedom are a formal device used to regulate the theory ${ }^{16}$. This means that correlators in both theories are those of the $G / G$ gauged WZW model, which is anomaly-free. These correlators have been studied in great detail [109,117,118]. In particular, careful discussions of the proper regularization of this theory can be found in these papers. As common in non-abelian gauge theories, to properly do these path integrals, one needs to introduce fermionic ghosts. One then finds a BRST charge $Q_{B R S T}$ obeying $\left(Q_{B R S T}\right)^{2}=0$; physical states are annihilated by it. We can thus consistently demand that states and physical operators be gauge invariant.

A relation between the $G / G$ gauged WZW model and topological field theory was conjectured in [109] and proven in [52]. In particular, it was shown that once we integrate over $A$ as well, the resulting partition function $|\chi|^{2}$ is independent of the metric of two-dimensional space. The correlators in the ground state therefore are independent of distance, and given by a topological field theory. A topological field theory is obtained by studying the states which are annihilated by $Q_{B R S T}$ but not given by $Q_{B R S T}$ acting on something else. In mathematical language, the physical states of the topological theory are given by the cohomology of $Q_{B R S T}$. It was derived directly in [123] that the

16 This approach is reminiscent of stone soup. 
correlators of the $G / G$ topological field theory are given in terms of the Verlinde numbers [108], which give the dimensions of conformal blocks in the ordinary WZW model.

Going to the doubled theory has therefore allowed us to not only avoid technical problems, but also to prove that the ground state correlators are those of a topological field theory. We should stress once again that the doubled theory used here has twice as many degrees of freedom as the undoubled theory. It is a physically distinct time-reversal invariant theory, unlike the undoubled theory which breaks timereversal symmetry. This physical difference is apparent from the construction of its observables: it has twice as many "anyons", which come in time-reversed pairs. On a torus, the Wilson loops of the corresponding topological field theory wind around both cycles [50]. The weights of the loops can be defined locally, and one can see how the non-abelian statistics arise [50]. The results of this section show how this precise topological field theory arises as the ground state of a specific Hamiltonian. We note in addition that if one specializes these results to an abelian gauge field, the topological field theory obtained is known as a (two-dimensional) BF theory [109, 124]. Recently, Freedman, Nayak and Shtengel [125] have discussed (reasonably local) lattice models of interacting fermions and bosons, which they argue have topological ground states with some of the topological algebraic structure of the doubled non-abelian Chern-Simons theories. It is reasonable to expect that the universal long-distance structure of the wave functions of these topological ground states, at least deep in the topologicallyordered phase, has the same structure of the wave functions we discussed in this section.

Since we have an explicit Hamiltonian Eq. (5.26) (plus the analogous term for the $B$ gauge field), we can go beyond the topological field theory describing the ground state. Because of the commutation relations (5.25), $E^{\dagger}$ acts like a creation operator. However, in a non-abelian gauge theory, $E$ and $E^{\dagger}$ are not gauge invariant, so the appropriate states are slightly more complicated than just $E^{\dagger} \Psi[A]$. The simplest candidate is [110]

$$
\psi^{a}(x, A, B)=E^{\dagger b}(x) \int[D g] \operatorname{Tr}\left[T^{a} g(x) T^{b} g^{-1}(x)\right] e^{-I(g, A, B)} .
$$

Once this amplitude is squared and $B$ is integrated over, one obtains a gauge-invariant probability. In the 2+1-dimensional picture, one can think of one of these states as a Polyakov loop, which intersects two-dimensional space at a single point $x$. The commutation relation Eq. (5.25) shows that this is indeed an eigenstate with a gap proportional to $k$. A more thorough treatment, taking into account the measure of the path integral, shows that the gap is shifted to $e^{2}\left(k+2 c_{A}\right) /(4 \pi)$, where $c_{A}$ is the quadratic Casimir of the adjoint representation of the Lie algebra of $G$ [11]. For our purposes, the important point is that there is indeed a gap.

We have thus seen that correlators of Wilson loops in the ground state of strongly-coupled YangMills theory are given by a topological field theory. We have also seen that the theory has a gap, so it is indeed in a topological phase. The last thing we would like to discuss is the ground-state correlators for operators not in the topological theory. In other words, we wish to consider the full set of physical operators (i.e. those annihilated by $Q_{B R S T}$ ), including those which are given by $Q_{B R S T}$ acting on something else. Because our Hamiltonian involves only the electric and not the magnetic field, it is not Lorentz invariant. Thus the existence of a gap does not immediately require that correlators decay exponentially. However, in this theory, they do, as implied by the results of [119]. We noted above that proper quantization of gauged WZW models requires introducing fermionic ghosts, and a fermionic operator $Q_{B R S T}$. A fermionic symmetry suggests the appearance of supersymmetry, and indeed the $G / G$ topological field theory can also be obtained from a supersymmetric field theory where the supersymmetry charge is "twisted" into $Q_{B R S T}$ 109 126 127, 128]. The supersymmetric field theory also describes the correlators of the full theory, not just the topological subsector. For the case where $G=S U(N)$ at level $k$, the appropriate two-dimensional field theory is a (twisted) supersymmetric sigma model, where the bosonic fields take values on the "Grassmannian" manifold $U(N+k) /(U(N) \times$ $U(k)$ [119]. It is likely that the other simple Lie groups end up giving supersymmetric sigma models on the analogous Grassmannians. 
This means we have now come full circle! We started without gauge fields, and found that the equal-time correlators are those of the principal chiral model, a two-dimensional sigma model with a curved target space. These correlators are exponentially decaying. We then introduced gauge fields, in the hope of finding a quantum critical point. After this lengthy discussion, we have ended up showing the ground state is described by a supersymmetric two-dimensional sigma model with a curved target space, the Grassmannian. This means that the correlators here are exponentially decaying as well, and the theory is in a topological phase whose properties are encoded in the wave function $\chi[A, B]$.

\section{Acknowledgments}

We are grateful to M.P.A. Fisher, M. Freedman, S. Kivelson, C. Nayak, K. Shtengel, T. Senthil, S. Sondhi, M. Stone, A. Vishwanath and X.G. Wen for many illuminating conversations on quantum dimer models, topological phases and beyond. We are grateful to M. Stone for motivating us to keep track of factors of $i$. We also thank M. Freedman, C. Nayak and Z. Wang for organizing a very stimulating conference on topological order at the American Institute of Mathematics, during which part of this work was done. This work was supported in part by the National Science Foundation through the grants NSF-DMR-01-32990 at the University of Illinois and NSF-DMR-0104799 at the University of Virginia. The work of P.F. was also supported by the DOE under grant DEFG0297ER41027.

\section{APPENDIX A: OPERATORS OF THE QUANTUM LIFSHITZ FIELD THEORY}

In addition to the products of field operators $\varphi(\vec{x})$, in what follows we will be interested in two types of local operators: charge and vortex operators. The charge operators are

$$
\mathcal{O}_{n}(\vec{x})=e^{-i n \varphi(\vec{x})}
$$

where $n \in \mathbb{Z}$. This operator creates a boson coherent-state which we will refer to as a charge $n$ excitation. The vortex operators are

$$
\tilde{\mathcal{O}}_{m}(\vec{x})=e^{i \int d^{2} z \varphi(\vec{z}) \Pi(\vec{z})}
$$

where

$$
\varphi(\vec{z})=m \arg (\vec{z}-\vec{x})
$$

where $0 \leq \arg (\vec{z}-\vec{x}) \leq 2 \pi$ is the argument of the vector $\vec{z}-\vec{x}$ (with a branch cut defined arbitrarily along the negative $x$ axis). The action of the operator $\tilde{\mathcal{O}}_{m}(\vec{x})$ on an eigenstate of the field operator $|[\varphi]\rangle$ is simply a shift

$$
e^{i \int d^{2} z \varphi(\vec{z}) \Pi(\vec{z})}|[\varphi]\rangle=|[\varphi(\vec{x})-\varphi(\vec{x})]\rangle
$$

In other words, it amounts to s singular gauge transformation. Therefore, its action is equivalent to coupling the field $\varphi$ to a vector potential whose space components $\vec{A}$ satisfy

$$
\oint_{\gamma} d \vec{z} \cdot \vec{A}[\vec{z}]=2 \pi m
$$


for all closed paths $\gamma$ which have the point $\vec{x}$ in their interior, and zero otherwise. In particular, the wave function of the state resulting from the action of the vortex operator on the ground state is:

$$
\Psi_{m}[\vec{x}]=\left\langle[\varphi]\left|\tilde{\mathcal{O}}_{m}(\vec{x})\right| \operatorname{vac}\right\rangle=\frac{1}{\sqrt{\mathcal{Z}}} e^{-\frac{\kappa}{2} \int d^{2} z(\vec{\nabla} \varphi-\vec{A})^{2}}
$$

where $\vec{A}$ is any vector field which satisfies Eq. (A5). The (equal-time) ground state expectation value of a product of vortex operators with magnetic charges $\left\{m_{l}\right\}$, i.e. the overlap of the state with $\mathrm{k}$ vortices at locations $\vec{x}_{l}$ and magnetic charge $m_{l}$ with the vortex-free ground state wave function, is therefore

$$
\left\langle\operatorname{vac}\left|\tilde{\mathcal{O}}_{m_{1}}\left(\vec{x}_{1}\right) \ldots \tilde{\mathcal{O}}_{m_{k}}\left(\vec{x}_{k}\right)\right| \operatorname{vac}\right\rangle=\frac{1}{\mathcal{Z}} \int \mathcal{D} \varphi e^{-\kappa \int d^{2} z(\vec{\nabla} \varphi-\vec{A})^{2}}
$$

where $\mathcal{Z}$ is given by Eq. (2.12). The vector potential in Eq. (A7) satisfies

$$
\varepsilon_{i j} \nabla_{i} A_{j}=2 \pi \sum_{l=1}^{k} m_{l} \delta^{2}\left(\vec{z}-\vec{x}_{l}\right)
$$

This result is equivalent to the expectation value of the vortex operator in the $2 \mathrm{D}$ classical $c=1$ compactified free bose field discussed extensively by Kadanoff [69] (see also [48]).

The boson propagator of this theory, in imaginary time $t$, is

$$
G\left(\vec{x}-\vec{x}^{\prime}, t-t^{\prime}\right)=\left\langle\varphi(\vec{x}, t) \varphi\left(\vec{x}^{\prime}, t^{\prime}\right)\right\rangle=\int \frac{d \omega}{2 \pi} \int \frac{d^{2} q}{(2 \pi)^{2}} \frac{e^{i \omega\left(t-t^{\prime}\right)-i \vec{q} \cdot\left(\vec{x}-\vec{x}^{\prime}\right)}}{\omega^{2}+\kappa^{2}\left(\vec{q}^{2}\right)^{2}}
$$

which has a short-distance logarithmic divergence. From now on we will use instead the regularized (subtracted) propagator

$$
G_{\mathrm{reg}}(\vec{x}, t) \equiv G(\vec{x}, t)-G(a, 0)=-\frac{1}{8 \pi \kappa}\left[\ln \left(\frac{|\vec{x}|^{2}}{a^{2}}\right)+\Gamma\left(0, \frac{|\vec{x}|^{2}}{4 \kappa|t|}\right)\right]
$$

where $a$ is a short-distance cutoff and $\Gamma(0, z)$ is the incomplete Gamma function

$$
\Gamma(0, z)=\int_{z}^{\infty} \frac{d s}{s} e^{-s}
$$

The regularized propagator has the asymptotic behaviors

$$
G_{\mathrm{reg}}(\vec{x}, t)= \begin{cases}-\frac{1}{4 \pi \kappa} \ln \left(\frac{|\vec{x}|}{a}\right), & \text { for }|t| \rightarrow 0 \\ -\frac{1}{8 \pi \kappa} \ln \left(\frac{4 \kappa|t|}{a^{2} \gamma}\right), & \text { for }|\vec{x}| \rightarrow a\end{cases}
$$

where $\ln \gamma=\mathbf{C}=0.577 \ldots$ is the Euler constant.

The correlation functions of the charge operators are

$$
\left\langle\mathcal{O}_{n}(\vec{x}, t)^{\dagger} \mathcal{O}_{n}\left(\vec{x}^{\prime}, t^{\prime}\right)\right\rangle=e^{n^{2}} G_{\mathrm{reg}}\left(\vec{x}-\vec{x}^{\prime}, t-t^{\prime}\right)
$$

At equal (imaginary) times, $\left|t-t^{\prime}\right| \rightarrow 0$, it behaves like

$$
\left\langle\mathcal{O}_{n}(\vec{x}, 0)^{\dagger} \mathcal{O}_{n}\left(\vec{x}^{\prime}, 0\right)\right\rangle=\left(\frac{a}{\left|\vec{x}-\vec{x}^{\prime}\right|}\right)^{\frac{n^{2}}{4 \pi \kappa}}
$$


which implies that the operator $\mathcal{O}_{n}$ has (spacial) scaling dimension

$$
\Delta_{n}=\frac{n^{2}}{8 \pi \kappa}
$$

For $\left|\vec{x}-\vec{x}^{\prime}\right| \rightarrow a$, its asymptotic behavior is instead given by

$$
\left\langle\mathcal{O}_{n}(\overrightarrow{0}, t)^{\dagger} \mathcal{O}_{n}\left(\overrightarrow{0}, t^{\prime}\right)\right\rangle=\left(\frac{a^{2} \gamma}{4 \kappa\left|t-t^{\prime}\right|}\right)^{\frac{n^{2}}{8 \pi \kappa}}
$$

This behavior is manifestly consistent with a dynamical critical exponent $z=2$.

It is straightforward to show by an explicit calculation of the overlap of Eq. (A8), which is completely analogous to the classical vortex correlation functions of the 2D Gaussian model [69], that the (spacial) scaling dimension of the vortex of magnetic charge $m$ is,

$$
\Delta_{m}=2 \pi \kappa m^{2}
$$

\section{APPENDIX B: $U(1)$ GAUGE-THEORY FOR THE QUANTUM SIX-VERTEX MODEL}

In this Appendix, we describe the quantum six-vertex model in the language of gauge theory. The quantum eight-vertex model will be described in appendix [C. We will follow closely the gauge theory description of the quantum dimer model, which is described in detail in [13]. We note that this gauge theory is not simply an abelian version of the gauge theory discussed in section $\nabla$

We define link variables $E_{i}(\vec{x})$, where $\vec{x}$ labels the vertices and $i=1,2$ indicates the direction; the unit vector in direction $i$ is denoted by $\vec{e}_{i}$. The link variables are integer valued, and can be viewed as the eigenvalues of angular momentum operators which we will also denote by $E_{i}(\vec{x})$. We assign the values $E_{1}=1$ and $E_{2}=1$ to the right and up going arrows respectively, while for the left and down going arrows we have $E_{1}=-1$ and $E_{2}=-1$. An example is given in figure 7 . In the Hamiltonian,

$$
\begin{array}{c|l}
E_{1}\left(\vec{x}-\vec{e}_{1}\right)=\underbrace{1}_{\vec{x}} & E_{2}(\vec{x})=1 \\
E_{2}\left(\vec{x}-\vec{e}_{2}\right)=-1 & E_{1}(\vec{x})=-1
\end{array}
$$

FIG. 7: The 'electric' fields associated to a particular vertex

we need a term which will lead to a restriction to those states in which all the $E_{i}(\vec{x})$ 's have values \pm 1 . Such a term is

$$
H_{\mathrm{E}}=\frac{1}{k_{1}} \sum_{\vec{x}, i}\left(E_{i}^{2}(\vec{x})-1\right)^{2}
$$

in the limit $k_{1} \rightarrow 0$.

The six-vertex model also requires that the same number of arrows point in and out at a vertex. This is precisely a lattice version of Gauss' law. Defining the lattice divergence as $\Delta_{i}^{-} E_{i}(\vec{x}) \equiv E_{1}(\vec{x})-$ $E_{1}\left(\vec{x}-\vec{e}_{1}\right)+E_{2}(\vec{x})-E_{2}\left(\vec{x}-\vec{e}_{2}\right)$, one easily finds that only for the six vertices of type $a, b$ and $c$, we have

$$
\Delta_{i}^{-} E_{i}(\vec{x})=0
$$


The lattice differentiation $\Delta_{i}^{-}$is defined by $\Delta_{i}^{-} f(\vec{x}) \equiv f(\vec{x})-f\left(\vec{x}-\vec{e}_{i}\right)$. In the following, when dealing with sums over the plaquettes of the lattice, we will frequently use $\Delta_{i} f(\vec{x}) \equiv \Delta_{i}^{+} f(\vec{x}) \equiv f\left(\vec{x}+\vec{e}_{i}\right)-f(\vec{x})$. Of course, the constraint has to commute with all the terms in the Hamiltonian. In the following, we will find that this is indeed the case.

The two main ingredients in the gauge theory description are the flip term which flips the flippable plaquettes (see below) and a potential term, which give a finite weight to (only) the flippable plaquettes. Flippable plaquettes are those which have both $n_{d}=\widetilde{n}_{d}=0$. Pictorially, a flippable plaquette here is one where the arrows around the plaquette point either all clockwise or all counterclockwise:

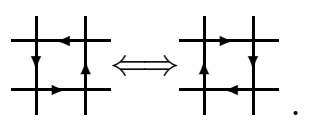

In terms of the electric field, this can be written as

$$
H_{\mathrm{V}}=\frac{V}{64} \sum_{\square}\left(\Delta_{2} E_{1}\right)^{2}\left(\Delta_{1} E_{2}\right)^{2}\left(E_{1}-E_{2}\right)^{2},
$$

where the sum is over all the plaquettes of the lattice. The factors $\left(\Delta_{2} E_{1}\right)^{2}$ and $\left(\Delta_{1} E_{2}\right)^{2}$ make sure that the arrows on opposite links of the plaquette are anti-parallel. The factor $\left(E_{1}-E_{2}\right)^{2}$ checks if two arrows on one vertex are both pointing clockwise or both counterclockwise.

As the $E_{i}(\vec{x})$ 's have the integers as their eigenvalues, the canonically conjugate operators, $a_{i}(\vec{x})$ are phases, i.e. $0 \leq a_{i}(\vec{x})<2 \pi$. Using the commutation relations

$$
\left[a_{j}(\vec{x}), E_{j^{\prime}}\left(\vec{x}^{\prime}\right)\right]=i \delta_{j j^{\prime}} \delta_{\vec{x}, \vec{x}^{\prime}}
$$

it is easy to show that the operators $e^{ \pm i a_{j}(\vec{x})}$ act as raising and lowering operators on the $E$ 's, and thus e.g. the operator $e^{-2 i a_{1}(\vec{x})}$ will flip the arrow pointing outward from $\vec{x}$ to the arrow pointing inward. We can use these raising and lowering operators to write the term in the Hamiltonian which flips the flippable plaquettes

$$
H_{\mathrm{t}}=-2 t \sum_{\square} \cos \left(2 \sum_{\square} a_{j}(\vec{x})\right)
$$

where $\sum_{\square} a_{j}(\vec{x})=\Delta_{1} a_{2}(\vec{x})-\Delta_{2} a_{1}(\vec{x})$ is the oriented sum of the $a$ 's around a plaquette. The total Hamiltonian of the gauge theory version of the 6 -vertex model is therefore

$$
H_{6 \mathrm{v}}=H_{\mathrm{E}}+H_{\mathrm{t}}+H_{\mathrm{v}}
$$

As usual, the Rokhsar-Kivelson point is located at $t=V$.

We will proceed by going to the dual formulation of the theory, and show that the theory is equivalent to a height model (which is well know). Doing the duality basically amounts to solving the (electrostatic) constraint. In the process, it gets replaced by a magnetic constraint. To solve the constraint, we introduce the new variables $S(\vec{r})$, which live on the sites of the dual lattice (or plaquettes of the direct lattice); these operators have the integers as their spectrum. In addition, we need the fields $B_{i}(\vec{r})$, which live on the links of the dual lattice. We can now write the "electric" fields $E_{i}$ as follows:

$$
E_{i}(\vec{x})=\epsilon_{i j}\left(\Delta_{j}^{-} S(\vec{r})+B_{j}(\vec{r})\right)
$$

Substituting this in the constraint $\Delta_{i}^{-} E_{i}=0$ gives the 'magnetic' constraint $\epsilon_{i j} \Delta_{j}^{-} B_{k}(\vec{r})=0$, so $B_{k}$ is curl free, and can be written as a gradient. But, as the there are no sources, we can do even better, as 
becomes clear when we interpret $S(\vec{r})$ as a height variable which lives on the plaquettes of the direct lattice.

We will first recall the known fact that the configurations of the 6-vertex model can be mapped onto height configurations. The rules are as follows. First, pick a reference site, and give it a reference height, say $S(\overrightarrow{0})=0$. Then, if one crosses an outgoing arrow clockwise (both seen from the vertex), the height decreases by one, while crossing an incoming arrow (again in a clockwise manner), the height increases by one. As all the vertices have two incoming and two outgoing arrows, this indeed gives a consistent height configuration. So, as an example, around an $a$ vertex we have

$$
\begin{array}{l|l}
1 & 0 \\
\hline 0 & \overrightarrow{-1}
\end{array} .
$$

We now assume that the $S(\vec{r})$ 's appearing in Eq. (B8) can in fact be interpreted as the heights of the plaquettes. Because of the duality Eq. (B8), the $B_{i}$ are now completely determined by the $E_{i}$, because they determine $S$ via the height rule. So we found that interpreting the $S(\vec{r})$ as heights amounts to picking a gauge for the $B_{i}(\vec{r})$. Combining the height rule of the previous paragraph with Eq. (B8), we easily find that $B_{j}(\vec{r}) \equiv 0$.

To complete the duality transformation, we need to transform the flip term. This will involve the canonically conjugate variable to $S(\vec{r})$. Let us call this the momentum $P(\vec{r})$, which satisfies $\left[P(\vec{r}), S\left(\vec{r}^{\prime}\right)\right]=i \delta_{\vec{r}, \vec{r}^{\prime}}$. Again, acting with $e^{i P(\vec{r})}$ on the plaquette at $\vec{r}$ will increase the eigenvalue of $S(\vec{r})$ by one. Flipping a plaquette changes $S$ by \pm 2 , so we find that we can write the flip term of the Hamiltonian as $H_{\mathrm{t}}=-2 t \sum_{\vec{r}} \cos (2 P(\vec{r}))$. In other words, we find that the circulation around the plaquette at $\vec{r}$ is given by $\sum_{\square} a_{j}(\vec{x})=P(\vec{r})$.

In principle, we could go on to describe the eight-vertex model in a similar fashion. The main difference with the six-vertex model is that the constraint $(\mathbb{B} 2)$ is no longer satisfied. One can introduce a matter field which has no-zero values on the $d$ vertices. In addition, a flip term which flips every plaquette has to be constructed. This flip term has to commute with the new constraint. It turns out that this is indeed possible, but the flip term will involve the conjugate of the matter field. Also, one would need a potential term which gives weights plaquettes according to the vertices present on the plaquette. However, as there is a more natural gauge description, which is based on the $\mathbb{Z}_{2}$ symmetry of the eight-vertex model, we will discuss and use that description of the quantum eight vertex model in the next appendix.

\section{APPENDIX C: A $\mathbb{Z}_{2}$ GAUGE THEORY FOR THE QUANTUM EIGHT VERTEX MODEL}

In this appendix, we will discuss a $\mathbb{Z}_{2}$ gauge theory which can be viewed as an extension of the Kitaev model which incorporates vertex weights differing from unity. The model we will discuss is of the Rokhsar-Kivelson type, but it is not the simplest quantum generalization of the classical eight-vertex model, as we pointed out in section [V]

\section{The $\mathbb{Z}_{2}$ gauge theory}

In this model, spins living on the bonds of the square lattice are the degrees of freedom. Thus, on every link of the square lattice, we define a Pauli algebra of $2 \times 2$ Hermitian matrices $\sigma_{j}^{a}(\vec{x})$, where $a=1,2,3$ labels the three Pauli matrices, and for a lattice site $\vec{x}$, we denoted the orientation of the link by $j=1,2(1$ =horizontal and $2=$ vertical). (Thus, the degrees of freedom live half-way between the lattice sites $\vec{x}$ and $\vec{x}+\vec{e}_{j}$, where $\vec{e}_{j}$ is a unit vector along the direction $j$.) In what follows we will take the states $|\uparrow\rangle$ (an "up" spin) and $|\downarrow\rangle$ ( a "down" spin) as the states which diagonalize $\sigma^{1}$ (instead of $\sigma^{3}$, as it is customary). The relation with the eight-vertex model is simple. Up-spins correspond 


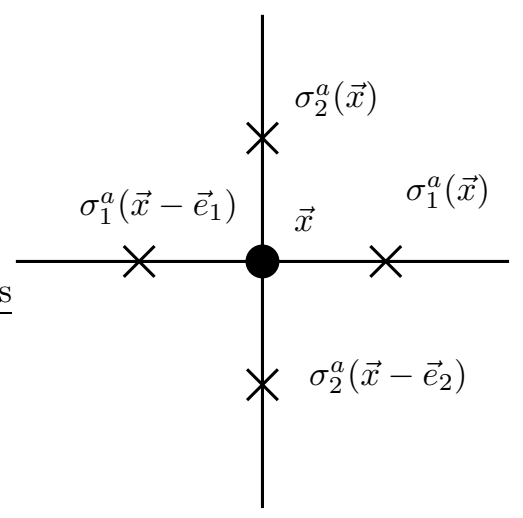

FIG. 8: Notation for the $\sigma$ 's; $a=1,2,3$ is the Pauli matrix label.

to arrows pointing up or to the right, while down spins correspond to arrows pointing down or to the left. Around each vertex, the number of up-spins has to be even. In this section, we will denote the vertex by $\vec{x}$ and the associated plaquette by its south-west corner $\vec{x}$.

For details, see Ref. [31,57]. The constraint can written in a simple form ${ }^{17}$

$$
\sigma_{1}^{1}(\vec{x}) \sigma_{1}^{1}\left(\vec{x}-\vec{e}_{1}\right) \sigma_{2}^{1}(\vec{x}) \sigma_{2}^{1}\left(\vec{x}-\vec{e}_{2}\right)=1, \quad \forall \vec{x}
$$

The term which flips all the arrows around a plaquette can be written in terms of $\sigma^{3}$ 's

$$
H_{\text {flip }}=-\sum_{\vec{x}} \sigma_{1}^{3}(\vec{x}) \sigma_{1}^{3}\left(\vec{x}+\vec{e}_{2}\right) \sigma_{2}^{3}(\vec{x}) \sigma_{2}^{3}\left(\vec{x}+\vec{e}_{1}\right)
$$

This flip operator commutes with the constraint, because a vertex and a plaquette have either 0 or 2 common bonds. Hence, both operators can be diagonalized simultaneously. In the model considered by Kitaev [42], equal weight is given to all types of vertices, so no term in the Hamiltonian is required. To go beyond the point $a=b=c=d=1$, we need a term which will weight the plaquettes according to the types of vertices present. Thus, we will need to introduce operators which can discern among the various vertices. In addition, the "weight" of the vertex depends on its position in the plaquette under consideration. The notation that we use is summarized in figure 8

Let us start by giving the terms which give a non-zero contribution for $a, b, c$ and $d$ vertices respectively (but zero otherwise). Let us define the "vertex magnetizations"

$$
\begin{aligned}
S_{a}(\vec{x}) & =\frac{1}{4}\left(\sigma_{1}^{1}(\vec{x})+\sigma_{2}^{1}(\vec{x})+\sigma_{1}^{1}\left(\vec{x}-\vec{e}_{1}\right)+\sigma_{2}^{1}\left(\vec{x}-\vec{e}_{2}\right)\right) \\
S_{b}(\vec{x}) & =\frac{1}{4}\left(\sigma_{1}^{1}(\vec{x})-\sigma_{2}^{1}(\vec{x})+\sigma_{1}^{1}\left(\vec{x}-\vec{e}_{1}\right)-\sigma_{2}^{1}\left(\vec{x}-\vec{e}_{2}\right)\right) \\
S_{c}(\vec{x}) & =\frac{1}{4}\left(\sigma_{1}^{1}(\vec{x})-\sigma_{2}^{1}(\vec{x})-\sigma_{1}^{1}\left(\vec{x}-\vec{e}_{1}\right)+\sigma_{2}^{1}\left(\vec{x}-\vec{e}_{2}\right)\right) \\
S_{d}(\vec{x}) & =\frac{1}{4}\left(\sigma_{1}^{1}(\vec{x})+\sigma_{2}^{1}(\vec{x})-\sigma_{1}^{1}\left(\vec{x}-\vec{e}_{1}\right)-\sigma_{2}^{1}\left(\vec{x}-\vec{e}_{2}\right)\right)
\end{aligned}
$$

With this notation, the projectors onto the vertices $a, b, c$ and $d$ are just the squares of the vertex magnetizations of Eq. (C3):

$$
\mathcal{P}_{a}=S_{a}^{2}, \quad \mathcal{P}_{b}=S_{b}^{2}, \quad \mathcal{P}_{c}=S_{c}^{2}, \quad \mathcal{P}_{d}=S_{d}^{2}
$$

\footnotetext{
17 Throughout we use the upper label to indicate the Pauli matrix and the lower label to indicate direction.
} 
It is straightforward to show that these operators act as projection operators on vertices of type $a, b$, $c$ and $d$ respectively, i.e. they yield 1 when acting on the corresponding vertex and zero otherwise. It is elementary to check that

$$
\mathcal{P}_{a}+\mathcal{P}_{b}+\mathcal{P}_{c}+\mathcal{P}_{d}=\mathcal{I}
$$

where $\mathcal{I}$ is the identity operator.

We can now write down the potential term which assigns weights to the plaquettes in the same way as done by the projectors of section [IV] Flipping a plaquette will change a $d$ vertex to an $a$ vertex if the $d$ vertex is in the south-west or north-east "even" corner of the plaquette. A $d$ at the other "odd" corners will go to a $b$ under the flip. In short, $d_{e} \leftrightarrow a_{e}, d_{o} \leftrightarrow b_{o}$. For $c$ 's this is opposite, namely $c_{e} \leftrightarrow b_{e}, c_{o} \leftrightarrow a_{o}$. The potential term will involve all four vertices around a plaquette, and thus we need to distinguish between the different position in the plaquette. This is achieved by the following, albeit rather cumbersome, term

$$
\begin{aligned}
H_{\mathrm{V}} & =\sum_{\vec{x}}\left(\frac{d}{a} \mathcal{P}_{a}(\vec{x})+\frac{c}{b} \mathcal{P}_{b}(\vec{x})+\frac{b}{c} \mathcal{P}_{c}(\vec{x})+\frac{a}{d} \mathcal{P}_{d}(\vec{x})\right) \times \\
& \left(\frac{c}{a} \mathcal{P}_{a}\left(\vec{x}+e_{1}\right)+\frac{d}{b} \mathcal{P}_{b}\left(\vec{x}+e_{1}\right)+\frac{a}{c} \mathcal{P}_{c}\left(\vec{x}+e_{1}\right)+\frac{b}{d} \mathcal{P}_{d}\left(\vec{x}+e_{1}\right)\right) \times \\
& \left(\frac{c}{a} \mathcal{P}_{a}\left(\vec{x}+e_{2}\right)+\frac{d}{b} \mathcal{P}_{b}\left(\vec{x}+e_{2}\right)+\frac{a}{c} \mathcal{P}_{c}\left(\vec{x}+e_{2}\right)+\frac{b}{d} \mathcal{P}_{d}\left(\vec{x}+e_{2}\right)\right) \times \\
& \left(\frac{d}{a} \mathcal{P}_{a}\left(\vec{x}+e_{1}+e_{2}\right)+\frac{c}{b} \mathcal{P}_{b}\left(\vec{x}+e_{1}+e_{2}\right)+\frac{b}{c} \mathcal{P}_{c}\left(\vec{x}+e_{1}+e_{2}\right)+\frac{a}{d} \mathcal{P}_{d}\left(\vec{x}+e_{1}+e_{2}\right)\right)
\end{aligned}
$$

The potential term (C6) assigns potential energies to the plaquettes in the same way as is done by the projectors of section IV] That is, the potential is the product of vertex weights obtained by flipping the plaquette, divided by the product of the vertex weights of the plaquette itself. As mentioned before, the two-body terms only couple spins on the same sublattice. The most non-local terms in the potential energy term consist of eight-body interactions. Of course, a plaquette potential energy term of this sort is needed, if one assigns weights to plaquettes ${ }^{18}$. Note that for $a=b=1$ and $c=d$ (or $c=\frac{1}{d}$ ), the eight-body terms cancel each other. However, there will remain four and six-body interactions, which will mix different sublattices.

The total Hamiltonian for the $\mathbb{Z}_{2}$ gauge theory of the quantum eight vertex model is

$$
H_{\mathrm{q} 8 \mathrm{v}}=H_{\mathrm{V}}+H_{\text {flip }}
$$

where the two terms are given by ([C2) and (C6). The states in this model have to satisfy the constraint (C1). We can check these formulas in a few limits. For $a=b=c=d=1$, each factor in (C6) will be 1 when acting on states satisfying the constraint. Hence, the potential term became the identity operator, which merely results in an energy shift, and thus we find back the Kitaev model, as we must.

Another interesting limit is $d \rightarrow 0$. As was discussed in section IVA this limit gives a slight generalization of the six-vertex model, in the sense that plaquettes which have $n_{d}=\widetilde{n}_{d}=1$ or $n_{d}=\widetilde{n}_{d}=2$ will have finite energy, and they can be interpreted as static defects. As can be seen from the potential term C6. plaquettes which have $n_{d}>\widetilde{n}_{d}$ will be suppressed as they receive infinite energy. The flip term has to be modified, because we need to have a flip term which commutes with the constraint, which has become a stronger statement in the six-vertex case, namely (c.f. Eq. (B2))

$$
\sigma_{1}^{1}(\vec{x})-\sigma_{1}^{1}\left(\vec{x}-\vec{e}_{1}\right)+\sigma_{2}^{1}(\vec{x})-\sigma_{2}^{1}\left(\vec{x}-\vec{e}_{2}\right)=0 \quad \forall \vec{x} .
$$

\footnotetext{
${ }^{18}$ In spite of the appearances, $H_{V}$ as given in Eq. C6 respects rotational invariance; the apparent asymmetry is due to the use of the vertex weights as labels.
} 
The flip term which preserves the six-vertex constraints is

$$
H_{\text {flip }, 6 \mathrm{v}}=-\sum_{\vec{x}}\left(\sigma_{1}^{-}(\vec{x}) \sigma_{2}^{-}\left(\vec{x}+e_{1}\right) \sigma_{1}^{+}\left(\vec{x}+e_{2}\right) \sigma_{2}^{+}(\vec{x})+\text { h.c. }\right) \text {, }
$$

where the raising and lowering operators (in the representation we use) are given by

$$
\sigma^{ \pm}=\frac{1}{2}\left(\sigma^{3} \mp i \sigma^{2}\right)
$$

To make contact with the flip term for the eight-vertex model, we rewrite Eq. (4.12) as

$$
\begin{aligned}
H_{\text {flip }, \mathrm{q} 8 \mathrm{v}}= & -\sum_{\vec{x}}\left(\sigma_{1}^{+}(\vec{x})+\sigma_{1}^{-}(\vec{x})\right)\left(\sigma_{2}^{+}\left(\vec{x}+e_{1}\right)+\sigma_{2}^{-}\left(\vec{x}+e_{1}\right)\right) \times \\
& \left(\sigma_{1}^{+}\left(\vec{x}+e_{2}\right)+\sigma_{1}^{-}\left(\vec{x}+e_{2}\right)\right)\left(\sigma_{2}^{+}(\vec{x})+\sigma_{2}^{-}(\vec{x})\right) .
\end{aligned}
$$

The flip term for the six-vertex model is therefore precisely the flip term for the eight-vertex model minus the terms which cause the six-vertex constraint to be violated. It is easily checked that the six-vertex flip term Eq. (C9) commutes with the constraint (C8). We thus find that on the level of the wave function, the limit $d \rightarrow 0$ is smooth, as the amplitude of the configurations which contain $d$ vertices goes to zero. In addition, for $d \neq 0$, the flip term commutes with constraint (C1), while for $d=0$, the flip term commutes with the $U(1)$ constraint (C8). Thus, the symmetry is enhanced from $\mathbb{Z}_{2}$ for $d \neq 0$ to $U(1)$ for $d=0$, as was to be expected.

\section{The dual of the gauge theory}

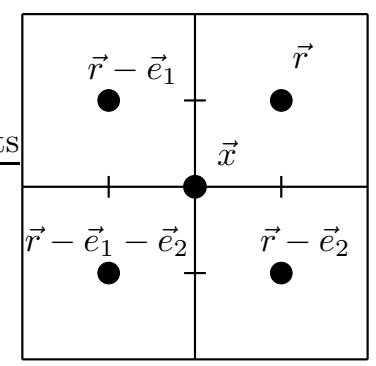

FIG. 9: The dual lattice sites are labeled by $\vec{r}$.

We now have a Rokhsar-Kivelson generalization of the eight-vertex model, in a gauge-theory language. We can use this representation of our model to study the various phases. However, this is more easily done in a dualized version, as the dual takes the form of an Ising model. In the dual picture, the spin degrees of freedom will live on the sites of dual square lattice, i.e. the centers of the plaquettes of the direct lattice. Thus, we will label by $\vec{r}$ the site of the dual lattice on the center of the plaquette labeled by $\vec{x}$ (its SW corner). Of course, the potential term in the dual language will still be quite formidable. We will denote the dual Pauli operators by $\tau^{1}$ and $\tau^{3}$. To start with the flip term, the product of $\sigma^{3}$ 's around a plaquette becomes $\tau^{1}$ on the plaquette [31,57]

$$
\tau^{1}(\vec{r})=\sigma_{1}^{3}(\vec{x}) \sigma_{2}^{3}\left(\vec{x}+\vec{e}_{1}\right) \sigma_{1}^{3}\left(\vec{x}+\vec{e}_{2}\right) \sigma_{2}^{3}(\vec{x})
$$

To see what happens with the constraint and the projector operators defined by Eq. (C3) and Eq. (C4) we need the dual form of the $\sigma^{1}$ 's living on the links. In term of the dual variables $\tau^{3}$, and using the notation of Fig. 9] the $\sigma^{1}$ 's are given by

$$
\sigma_{1}^{1}(\vec{x})=\tau^{3}(\vec{r}) \tau^{3}\left(\vec{r}-\vec{e}_{2}\right), \quad \sigma_{2}^{1}(\vec{x})=\tau^{3}(\vec{r}) \tau^{3}\left(\vec{r}-\vec{e}_{1}\right)
$$


We thus easily find that the constraint is automatically satisfied (again, going to the dual picture amounts to solving the constraint). Also, it is trivial to show [31,57] that the inverse relation, i.e. to express the dual lattice $\tau^{3}$ operators in terms of the $\sigma^{1}$ operators of the original lattice is

$$
\tau^{3}(\vec{r})=\prod_{\ell \in \Gamma(\vec{r})} \sigma^{1}(\ell)
$$

where $\{\ell\}$ is a set of links of the direct lattice pierced by a path $\Gamma(\vec{r})$ on the dual lattice ending at the dual site $\vec{r}$ (but which is otherwise arbitrary); see Fig. 10.

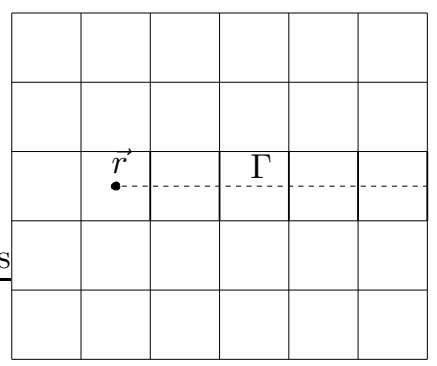

FIG. 10: The dual path $\Gamma$.

Note that we need to choose the spin on one of the plaquettes; all the others are subsequently determined by the $\sigma^{x}$ 's on the links. In terms of the dual variables, the projection operators for site $\vec{x}$, defined by Eq. (C4), take the form

$$
\begin{aligned}
& \mathcal{P}_{a}(\vec{x})=\frac{1}{4}(1+\mathcal{A}(\vec{r})+\mathcal{B}(\vec{r})+\mathcal{C}(\vec{r})) \\
& \mathcal{P}_{b}(\vec{x})=\frac{1}{4}(1-\mathcal{A}(\vec{r})-\mathcal{B}(\vec{r})+\mathcal{C}(\vec{r})) \\
& \mathcal{P}_{c}(\vec{x})=\frac{1}{4}(1-\mathcal{A}(\vec{r})+\mathcal{B}(\vec{r})-\mathcal{C}(\vec{r})) \\
& \mathcal{P}_{d}(\vec{x})=\frac{1}{4}(1+\mathcal{A}(\vec{r})-\mathcal{B}(\vec{r})-\mathcal{C}(\vec{r})),
\end{aligned}
$$

where $\mathcal{A}, \mathcal{B}$ and $\mathcal{C}$ are given by

$$
\begin{aligned}
& \mathcal{A}(\vec{r})=\tau^{3}\left(\vec{r}-\vec{e}_{1}\right) \tau^{3}\left(\vec{r}-\vec{e}_{2}\right) \\
& \mathcal{B}(\vec{r})=\tau^{3}(\vec{r}) \tau^{3}\left(\vec{r}-\vec{e}_{1}-\vec{e}_{2}\right) \\
& \mathcal{C}(\vec{r})=\tau^{3}(\vec{r}) \tau^{3}\left(\vec{r}-\vec{e}_{1}\right) \tau^{3}\left(\vec{r}-\vec{e}_{1}\right) \tau^{3}\left(\vec{r}-\vec{e}_{1}-\vec{e}_{2}\right) .
\end{aligned}
$$

The interaction in terms of these projectors has the same structure as in the spin representation of the classical eight-vertex model: it consists of two-body terms on interpenetrating sublattices, and a four-body term coupling the two sublattices. All the two-body interaction terms will only couple spins on the same sublattice. The four- and six-body interaction terms (of which there are many!), couple the sublattices. The same holds for the eight-body term, naturally. The dual form of the theory is

$$
H_{\mathrm{q} 8 \mathrm{v}, \mathrm{dual}}=H_{\mathrm{V}, \text { dual }}-\sum_{\vec{r}} \tau^{1}(\vec{r})
$$

where $H_{\mathrm{V} \text {,dual }}$ is given by (C6), but now with the projectors given in Eq. (C15). Thus, formally this theory takes the form of a (multi-spin) Ising model in a transverse field. However, the two-body interactions only couple spins on the same sublattices, together with the multi-spin terms conspire to 
change the quantum critical behavior from the conventional $z=1$ Lorentz-invariant criticality of the standard Ising model in a transverse field to the $z=2$ quantum critical behavior discussed in the rest of this paper.

Now that we found the dual version of our gauge theory, we would like to discuss the limits $a=b=c=d=1$ and $d=0$. Again, the first limit brings us back to the Kitaev point, because the potential term becomes the identity operator again, and we are left with the very simple spin flip term of Eq. (C17), $H_{\mathrm{f}}=-\sum_{\vec{r}} \tau^{1}(\vec{r})$. The limit $d \rightarrow 0$ is however more complicated in this dual gauge theory. First of all, we now do need a constraint, which was not present for $d \neq 0$. Moreover, the flip term now only can act, depending on the surrounding spins. Let us start by dualizing the constraint Eq. (C8), which results in

$$
\left(\tau^{3}(\vec{r})-\tau^{3}\left(\vec{r}-\vec{e}_{1}-\vec{e}_{2}\right)\right)\left(\tau^{3}\left(\vec{r}-\vec{e}_{1}\right)+\tau^{3}\left(\vec{r}-\vec{e}_{2}\right)\right)=0 \quad \forall \vec{x} .
$$

Obviously, the eight-vertex flip term $\tau^{1}(\vec{r})$ does not commute with this constraint. To find a flip term which does commute with the constraint, we dualize the six-vertex flip term (C9), which results in

$H_{\text {flip,q6v }}=-\frac{1}{8} \sum_{\vec{r}} \tau^{1}(\vec{r})\left(1-\tau^{3}\left(\vec{r}+\vec{e}_{1}\right) \tau^{3}\left(\vec{r}+\vec{e}_{2}\right)\right)\left(1+\tau^{3}\left(\vec{r}-\vec{e}_{1}\right) \tau^{3}\left(\vec{r}+\vec{e}_{2}\right)\right)\left(1+\tau^{3}\left(\vec{r}+\vec{e}_{1}\right) \tau^{3}\left(\vec{r}-\vec{e}_{2}\right)\right)$.

The factors in bracket can be seen to give a non zero result only on plaquettes which are flippable. Hence, this flip term commutes with the constraint (C18). Of course, this can also be checked explicitly. Apart from a factor $\tau^{2}$, there are factors depending on the $\tau^{3}$ 's coming form both the flip term and the constraint. The signs in this product conspire in such a way to render the commutator zero.

* Electronic address: ardonne@uiuc.edu

$\dagger$ Electronic address: fendley@virginia.edu

$\ddagger$ Electronic address: efradkin@uiuc.edu

1 S. A. Kivelson, Synthetic Metals 125, 99 (2002) arXiv:cond-mat/0106126, a recent review on the current status of electron fractionalization in Condensed Matter Physics.

2 P. Chaikin and T. C. Lubensky, Principles of Condensed Matter Physics (Cambridge University Press, Cambridge, UK, 1995).

3 J. Cardy, Scaling and Renormalization in Statistical Physics (Cambridge University Press, Cambridge, UK, 1996).

4 R. E. Prange and S. M. Girvin, eds., The Quantum Hall Effect (Springer-Verlag, 1987).

5 X. G. Wen, Phys. Rev. B 41, 12838 (1990).

6 X. G. Wen and Q. Niu, Phys. Rev. B 41, 9377 (1990).

7 T. Senthil and M. P. A. Fisher, Phys. Rev. B 62, 7850 (2000) arXiv:cond-mat/9910224.

8 R. Moessner and S. L. Sondhi, Phys. Rev. Lett. 86, 1881 (2001) arXiv:cond-mat/0007378.

9 S. A. Kivelson, D. Rokhsar, and J. Sethna, Phys. Rev. B 35, 865 (1987).

10 D. Rokhsar and S. A. Kivelson, Phys. Rev. Lett. 61, 2376 (1988).

11 P. Fendley, R. Moessner, and S. L. Sondhi, Phys. Rev. B 66, 214513 (2002) arXiv:cond-mat/0206159.

12 E. Fradkin, in Field Theories in Condensed Matter Physics, a Workshop, edited by Z. Tesanovic (Addison Wesley, Redwood City, 1990), Proceedings of the Johns Hopkins Workshop on Field Theories in Condensed Matter Physics.

13 E. Fradkin, Field Theories of Condensed Matter Systems (Addison-Wesley, Redwood City, 1991), see Chapter 6 .

14 R. Moessner, S. L. Sondhi, and E. Fradkin, Phys. Rev. B 65, 024504 (2002) arXiv:cond-mat/0103396.

15 P. W. Anderson, Science 235, 1196 (1987).

16 G. Baskaran and P. W. Anderson, Phys. Rev. B 37, 580 (1988).

17 I. Affleck and J. Marston, Phys. Rev. B 37, 3774 (1988).

18 G. Kotliar, Phys. Rev. B 37, 3664 (1988).

19 L. B. Ioffe and A. I. Larkin, Phys. Rev. B 40, 6941 (1989). 
20 P. A. Lee, N. Nagaosa, T.-K. Ng, and X.-G. Wen, Phys. Rev. B 57, 6003 (1998), arXiv:cond-mat/9701168, and references therein.

21 V. Kalmeyer and R. Laughlin, Phys. Rev. Lett. 59, 2095 (1988).

22 X.-G. Wen, F. Wilczek, and A. Zee, Phys. Rev. B 39, 11413 (1989).

23 N. Read and S. Sachdev, Phys. Rev. B 42, 4568 (1990).

24 C. Mudry and E. Fradkin, Phys. Rev. B 49, 5200 (1994) arXiv:cond-mat/9309021.

25 E. Fradkin and S. H. Shenker, Phys. Rev. D 19, 3682 (1979).

26 E. Witten, Commun. Math. Phys. 121, 351 (1989).

27 J. Preskill and P. Krauss, Nucl. Phys. B 341, 50 (1990).

28 M. de Wild Propitius, Topological Interactions in Broken Gauge Theories (1995), Ph.D. Thesis, University of Amsterdam; arXiv:hep-th/9511195.

29 A. M. Polyakov, Gauge Fields and Strings (Harwood Academic Publishers, London, 1987).

30 G. 't Hooft, Nucl. Phys. B 138, 1 (1978).

31 E. Fradkin and L. Susskind, Phys. Rev. D 17, 2637 (1978).

32 L. P. Kadanoff and H. Ceva, Phys. Rev. B 3, 3918 (1971).

33 S. Chakravarty, R. B. Laughlin, D. K. Morr, and C. Nayak, Phys. Rev. B 63, 094503 (2001) arXiv:cond-mat/0005443.

34 S. Kivelson, E. Fradkin, and V. Emery, Nature 393, 550 (1998).

35 S. Sachdev, Rev. Mod. Phys. 75, 913 (2003) arXiv:cond-mat/0211005.

36 R. Moessner and S. L. Sondhi, Phys. Rev. B 63, 224401 (2001) arXiv:cond-mat/0011250.

37 L. Balents, M. P. A. Fisher, and S. M. Girvin, Phys. Rev. B 65, 224412 (2002) arXiv:cond-mat/0110005.

38 T. Senthil and O. I. Motrunich, Phys. Rev. Lett. 89, 277004 (2002) arXiv:cond-mat/0205170.

39 T. Senthil, A. Vishwanath, L. Balents, S. Sachdev, and M. P. A. Fisher (2003), arXiv: cond-mat/0311326

40 A. Shapere and F. Wilczek, eds., Geometric phases in physics (World-Scientific, 1989).

41 G. Moore and N. Read, Nucl. Phys. B 360, 362 (1991).

42 A. Y. Kitaev, Annals of Physics 303, 2 (2003) arXiv:quant-ph/9707021.

43 M. H. Freedman (2001), arXiv:quant-ph/0110060.

44 M. H. Freedman, A. Kitaev, M. J. Larsen, and Z. Wang, arXiv:quant-ph/0101025.

45 D. Beckman, D. Gottesman, A. Kitaev, and J. Preskill, Phys. Rev. D 65, 065022 (2002) arXiv:hep-th/0110205.

46 B. Douçot, L. Ioffe, and J. Vidal, arXiv:cond-mat/0302104.

47 S. Sachdev, Quantum Phase Transitions (Cambridge University Press, Cambridge, England, 1999).

48 P. D. Francesco, P. Mathieu, and D. Sénéchal, Conformal Field Theory (Springer-Verlag, New York, 1997).

49 G. Baym, Lectures on Quantum Mechanics (Addison Wesley, Reading, MA, 1990).

50 M. Freedman, C. Nayak, K. Shtengel, K. Walker, and Z. Wang (2003), arXiv: cond-mat/0307511

51 T. H. Hansson, V. Oganesyan, and S. L. Sondhi (2003), unpublished.

52 E. Witten, Commun. Math. Phys. 144, 189 (1992).

53 C. L. Henley, J. Stat. Phys. 89, 483 (1997) arXiv:cond-mat/9607222.

54 G. Grinstein, Phys. Rev. B 23, 4615 (1981).

55 M. Henkel, Nucl. Phys. B 641, 405 (2002).

56 M. Henkel, A. Picone, M. Pleimling, and J. Unterberger, in Progress in Mathematical Physics (Nova Science, New York, 2003).

57 J. B. Kogut, Rev. Mod. Phys. 51, 659 (1979).

58 D. P. Arovas and S. M. Girvin (1991), Lectures presented at the Seventh International Conference on Recent Progress in Many Body Theories, Minneapolis, 26-30 August 1991.

59 K. Symanzik, Nucl. Phys. B 190, 1 (1981).

60 K. Symanzik, in Structural Elements in Particle Physics and Statistical Mechanics, edited by J. Honerkamp, K. Pohlmeyer, and H. Romer (Plenum Press, New York, 1983), p. 287, Proceedings of the NATO Advanced Summer Institute on Theoretical Physics, 1981.

61 E. Fradkin, Nucl. Phys. B 389, 587 (1993).

62 E. Fradkin, E. F. Moreno, and F. A. Schaposnik, Nucl. Phys. B 392, 667 (1993) arXiv:cond-mat/9905192.

63 B. Sutherland, J. Math. Phys. 12, 246 (1971).

64 F. D. M. Haldane, Phys. Rev. Lett. 60, 635 (1988).

65 B. S. Shastry, Phys. Rev. Lett. 60, 639 (1988).

66 M. E. Fisher and J. Stephenson, Phys. Rev 132, 1411 (1963).

67 S. Samuel, J. Math. Phys. 21, 2806 (1980).

68 F. A. Smirnov, Form Factors in Completely Integrable Models (World Scientific, Singapore, 1992). 
69 L. P. Kadanoff, Annals of Physics (N.Y.) 120, 39 (1979).

70 E. Fradkin and S. A. Kivelson, Mod. Phys. Lett. B 4, 225 (1990).

71 N. Read and S. Sachdev, Phys. Rev. Lett. 62, 1694 (1989).

72 W. Zheng and S. Sachdev, Phys. Rev. B 40, 2704 (1989).

73 L. S. Levitov, Phys. Rev. Lett. 64, 92 (1990).

74 V. Elser, Phys. Rev. Lett. 62, 2305 (1989).

75 A. Vishwanath, L. Balents, and T. Senthil (2003), arXiv:cond-mat/0311085.

76 E. Fradkin, D. Huse, R. Moessner, V. Oganesyan, and S. L. Sondhi (2003), arXiv:cond-mat/0311353.

77 J. Kondev and C. L. Henley, Phys. Rev. Lett. 73, 2786 (1994).

78 S. Sachdev and K. Park, Annals of Phys. (N. Y.) 298, 58 (2002), arXiv:cond-mat/0108214.

79 R. Jalabert and S. Sachdev, Mod. Phys. Lett. 4, 1043 (1990).

80 G. Grinstein and C. Jayaprakash, Phys. Rev. B 25, 523 (1982).

81 G. Grinstein and R. A. Pelcovits, Phys. Rev. Lett. 47, 856 (1981).

82 P. G. de Gennes and J. Prost, The Physics of Liquid Crystals (Oxford Science Publications, New York, 1998).

83 D. S. Fisher and J. D. Weeks, Phys. Rev. Lett. 50, 1077 (1983).

84 E. Fradkin, Phys. Rev. B 28, 5338 (1983).

85 R. Baxter, Exactly Solved Models in Statistical Mechanics (Academic Press, 1982), and references therein.

86 C. Fan and F. Y. Wu, Phys. Rev. B 2, 723 (1970).

87 G. Misguich, D. Serban, and V. Pasquier, Phys. Rev. Lett. 89, 137202 (2002) arXiv:cond-mat/0204428.

88 R. Moessner and S. L. Sondhi (2003) cond-mat/0212363.

89 S. Chakravarty, Phys. Rev. B 66, 224505 (2002) arXiv:cond-mat/0206282.

90 B. Douçot, M. Feigel'man, and L. Ioffe, arXiv:cond-mat/0211146.

91 M. Hermele, M. P. A. Fisher, and L. Balents (2003), arXiv: cond-mat/0305401

92 A. E. Rana and S. M. Girvin, Phys. Rev. B 48, 360 (1993) arXiv:cond-mat/9301023.

93 R. Moessner, O. Tchernyshyov, and S. L. Sondhi (2001), arXiv:cond-mat/0106286.

94 L. P. Kadanoff, Phys. Rev. Lett. 39, 903 (1977).

95 L. P. Kadanoff and A. C. Brown, Annals of Physics (N. Y.) 121, 318 (1979).

96 L. Dixon, D. Friedan, E. Martinec, and S. Shenker, Nucl. Phys. B 282, 13 (1987).

97 E. Fradkin and S. Raby, Phys. Rev. D 20, 2566 (1979).

98 T. Senthil and M. P. A. Fisher, Phys. Rev. B 63, 134521 (2001) arXiv:cond-mat/0006481.

99 P. Ginsparg, in Champs, Cordes et Phénoménes Critiques/ Fields, Strings and Critical Phenomena, edited by E. Brézin and J. Zinn-Justin (Elsevier Science Publishers B.V., 1989), Lectures given at Les Houches summer session, June 28 - Aug. 5, 1988; Les Houches, Session XLIX, 1988.

100 M. E. Peskin, Phys. Lett. B 94, 161 (1980).

101 J. M. Kosterlitz and D. J. Thouless, J. Phys. C 6, 1181 (1973).

102 J. M. Kosterlitz, J. Phys. C 7, 1046 (1974).

103 V. L. Berezinskii, ZhETF(USSR) 59, 907 (1970), Sov. Phys. JETP 32, 493 (1971).

104 J. Jose, L. P. Kadanoff, S. Kirkpatrick, and D. R. Nelson, Phys. Rev. B 16, 1217 (1977).

105 D. J. Amit, Field Theory, the Renormalization Group, and Critical Phenomena (World Scientific, Singapore, Singapore, 1984), second edition.

106 E. Witten, Commun. Math. Phys. 92, 455 (1984).

107 S. Elitzur, G. W. Moore, A. Schwimmer, and N. Seiberg, Nucl. Phys. B 326, 108 (1989).

108 E. Verlinde, Nucl. Phys. B 300, 360 (1988).

109 M. Spiegelglas, Phys. Lett. B 274, 21 (1992), M. Spiegelglas and S. Yankielowicz, Nucl. Phys. B 393, 301 (1993) arXiv:hep-th/9201036.

110 G. Grignani, G. W. Semenoff, P. Sodano, and O. Tirkkonen, Nucl. Phys. B 489, 360 (1997) arXiv:hep-th/9609228.

111 D. Karabali, C. J. Kim, and V. P. Nair, Nucl. Phys. B 566, 331 (2000) arXiv:hep-th/9907078.

112 X. G. Wen, Phys. Rev. B 44, 2664 (1991).

113 X. G. Wen, Adv. Phys. 44, 405 (1995) arXiv:cond-mat/9506066.

114 G. Dunne, R. Jackiw, and C. Trugenberger, Annals of Phys. 194, 197 (1989).

115 A. M. Polyakov and P. B. Wiegmann, Phys. Lett. B 131, 121 (1983).

116 S. Deser, R. Jackiw, and S.Templeton, Annals of Phys. (N. Y.) 140, 372 (1982).

117 K. Gawedzki and A. Kupiainen, Nucl. Phys. B 320, 625 (1989).

118 D. Karabali and H. J. Schnitzer, Nucl. Phys. B 329, 649 (1990).

119 E. Witten, in Geometry, topology, and physics, Cambridge 1993 (1993) p. 357, arXiv:hep-th/9312104. 
120 X. G. Wen, Phys. Rev. B 60, 8827 (1999) arXiv:cond-mat/9811111.

121 E. Fradkin, C. Nayak, and K. Schoutens, Nucl. Phys. B 546, 711 (1999) arXiv:cond-mat/9811005.

122 D. C. Cabra, E. Fradkin, G. L. Rossini, and F. A. Schaposnik, Int. J. Mod. Phys. A 30, 4857 (2000) arXiv:cond-mat/9905192.

123 A. Gerasimov (1993), Localization in GWZW and the Verlinde formula; arXiv:hep-th/9305090

124 G. L. Rossini and F. A. Schaposnik, Mod. Phys. Lett. A 9, 193 (1994) hep-th/9210084.

125 M. Freedman, C. Nayak, and K. Shtengel (2003), arXiv:cond-mat/0309120.

126 D. Gepner, Commun. Math. Phys. 141, 381 (1991).

127 C. Vafa, in Mirror symmetry, edited by S. T. Yau (1991), p. 97, arXiv:hep-th/9111017.

128 K. A. Intriligator, Mod. Phys. Lett. A 6, 3543 (1991). 